\title{
Nanophotonic devices for optical interconnect
}

\author{
Dries Van Thourhout, Member, IEEE, Thijs Spuesens, Student Member, IEEE, Shankar Kumar \\ Selvaraja, Student Member, IEEE, Liu Liu, Member, IEEE, Günther Roelkens, Member, IEEE, Rajesh \\ Kumar, Geert Morthier, Senior Member, IEEE, Pedro Rojo-Romeo, Member, IEEE, Fabien Mandorlo, \\ Philippe Régreny, Oded Raz, Christophe Kopp, Laurent Grenouillet
}

\begin{abstract}
We review recent progress in nanophotonic devices for compact optical interconnect networks. We focus on microdisk laser based transmitters and discuss improved design and advanced functionality including all-optical wavelength conversion and flip-flops. Next we discuss the fabrication uniformity of the passive routing circuits and their thermal tuning. Finally we discuss the performance of a wavelength selective detector.
\end{abstract}

Index Terms - Photonic integration, Silicon-on-Insulator, Heterogeneous Integration, Microdisk Laser, Network-on-Chip, Flip-Flop, Wavelength Conversion, Process Uniformity

\section{INTRODUCTION}

W ith the required data rates ever increasing optical communication is coming closer and closer to the chip. To be a valid alternative for electrical communication, the optical link has to provide at least the same bandwidth as current copper solutions at lower cost and with improved density. Current short reach optical interconnect solutions are mostly based on multimode fibers and/or waveguides in combination with VCSELs [1,2]. These do not provide a long term solution for the bandwidth density problem however. The line rates are limited and the currently proposed systems often require costly assembly techniques because they are not compatible with wafer scale fabrication.

Silicon photonics potentially could provide optical links, which are scalable in bandwidth, e.g. by using wavelength division multiplexing (WDM) and which are compatible with waferscale fabrication and testing. Recently it was shown by

Manuscript submitted September 1, 2009.

This work was supported by the European Commission through projects ICT-WADIMOS and ICT-HISTORIC under the 7th Framework Programme (FP7), Information and Communications Technologies (ICT).

D. Van Thourhout, T. Spuesens, S. K. Selvaraja, L. Liu, G. Roelkens, R. Kumar and G. Morthier are with INTEC department, Ghent University-IMEC, St-Pietersnieuwstraat 41, 9000 Ghent, Belgium (L. Liu is now with Department of Photonics Engineering, Technical University of Denmark, DTU - Fotonik, DK-2800 Kongens Lyngby, Denmark) (email: dries.vanthourhout@intec.ugent.be)

P. Rojo-Romeo, F. Mandorlo and P. Régreny are with Institut des Nanotechnologies de Lyon INL-UMR5270, CNRS, Université de Lyon, Ecole Centrale de Lyon, Ecully F-69134, France

O. Raz is with Eindhoven University of Technology, Den Dolech 2, $5600 \mathrm{MB}$, Eindhoven, The Netherlands

C. Kopp and L. Grenouillet are with CEA-LETI, Minatec 17 rue des Martyrs, 308054 Grenoble, France. several groups that it is possible to fabricate very compact optical waveguide circuits using the same type of equipment and tools as those used for fabricating the most advanced electronic integrated circuits [3-5]. The waveguides are fabricated starting from a silicon-on-insulator substrate with a $200 \mathrm{~nm}-250 \mathrm{~nm}$ thick silicon top layer, on which the devices are patterned using either electron beam lithography or deep UV lithography. Subsequently the waveguides are defined using reactive ion etching. Due to the high index contrast a very small bend radius can be used $\left(0.01 \mathrm{~dB} / 90^{\circ}\right.$ loss for a $5 \mu \mathrm{m}$ bend, $0.04 \mathrm{~dB} / 90^{0}$ loss for a $2 \mu \mathrm{m}$ bend). However, also because of the high index contrast, even the smallest remaining sidewall roughness - published values range from 1 to $3 \mathrm{~nm}$ - gives rise to considerable propagation losses $(1-3 \mathrm{~dB} / \mathrm{cm})$. Thanks to the compactness of the devices, the propagation loss often has only a minor impact on their overall performance however. Several groups now also demonstrated active devices such as modulators [6-8] and high speed photodetectors integrated with such waveguide circuits [9]. When using an off-chip source, these devices in principle provide sufficient functionality for building data links [10] or even more complex optical networks [11-13]. Further improvement in power consumption and size of these components is still required though [14].

For complex optical networks, using an off chip optical source becomes increasingly difficult however. The circuits required for distributing the optical feed may induce considerable loss, consume an important fraction of the available space and are difficult to design. Therefore we investigated the possibility for integrating compact micro sources directly on top of the electronic circuits. The requirements for these micro sources are very stringent. They should have low power consumption and compact footprint, they should be electrically injected and compatible with direct modulation at 5 to $10 \mathrm{~Gb} / \mathrm{s}$ and they should couple the light efficiently to the silicon optical waveguide layer. In addition, they should be compatible with waferscale fabrication technologies. In section II of this paper we describe the micro disk laser we developed for this purpose and its basic properties (threshold, spectrum, direct modulation). In section III, we describe some advanced properties, which may be necessary for scaling up wavelength routed networks [15], such as all optical wavelength conversion, all-optical gating and optical bistability. In section IV we give some more details on the fabrication and characteristics of the passive waveguide circuits and in particular on the fabrication uniformity and tolerances. We also propose integrated thermal tuners, which may help to overcome the device non-uniformity. In section $\mathrm{V}$ we describe the development of a wavelength selective detector. 


\section{MICRODISK LASER BASED TRANSMITTER}

\section{A. Introduction}

Thus far, only compound semiconductor materials with a direct bandgap have shown to exhibit sufficient gain for realizing compact electrically driven micro sources. Unfortunately, due to the large lattice mismatch, monolithic integration with silicon has proven to be difficult. Hybrid integration, whereby prefabricated components are integrated with the waveguides using accurate pick-and-place equipment does allow for prescreening of the lasers but is not compatible with wafer scale integration and hence not suitable for use in combination with very dense optical network circuits [16-18]. Therefore, several research groups proposed and demonstrated an alternative integration process based on the use of wafer and/or die bonding [19-21]. Thereby high quality InP-based epitaxial layers are bonded on-top of preprocessed silicon waveguides. Direct bonding processes [22] as well as molecular (silica-to-silica) [23] and adhesive bonding processes have been used [24]. Subsequently the substrate is removed and the optoelectronic devices are defined collectively using waferscale processes.

The microsources we developed were fabricated using this so-called heterogeneous integration process. Because they had proven to be efficient light emitters already using a monolithic integration platform [25], we decided to focus on microdisk type devices. Initial devices were optically pumped $[26,27]$ or not yet coupled to silicon waveguides [28]. In 2007 we demonstrated for the first time an electrically injected microlaser [29]. Continuous wave operation (CW) could be shown but the optical output power and external efficiency were still low. Recently we demonstrated a new generation of devices with improved epitaxial layers and processing scheme, leading to a considerable decrease in threshold current and the demonstration of new functionality such as bistable operation (see section III). In the next section, we describe these design improvements in more detail.

\section{B. Improved micro disk laser design and operation}

Fig. 1 gives a schematic representation of the heterogeneously integrated microdisk laser. The device was etched in a thin InP-based membrane, which was bonded on top of the silicon waveguides using an adhesive bonding process based on Benzocyclobutene (BCB). The silicon waveguide underneath was $500 \mathrm{~nm}$ wide and had a thickness of $220 \mathrm{~nm}$ (see [30] for details on the fabrication process). The laser mode is evanescently coupled to this waveguide. Careful alignment of the disk with respect to the waveguide is important to achieve a good coupling efficiency (see [31] for details on the disk-waveguide coupling design). Electrical injection is possible via a top metal contact in the center of the disk and a bottom contact on a $100 \mathrm{~nm}$ thin lateral contact layer. The details of the layer structure are shown in Table 1.

The heterostructures were grown at $490^{\circ} \mathrm{C}$ using solid source molecular beam epitaxy (SSMBE) on a two inch InP wafer supplied by InPact S.A. An InGaAs sacrificial etch-stop layer, which is removed following bonding, was grown first. The structure consists, starting from the bottom layer, of a $95 \mathrm{~nm}$ thick $5 \times 10^{18} \mathrm{~cm}^{-3}$ Si doped InP bottom contact layer, a $120 \mathrm{~nm}$ thick InGaAsP quaternary emitting at $1.2 \mu \mathrm{m}(\mathrm{Q} 1.2) 1 \times 10^{18}$ $\mathrm{cm}^{-3} \mathrm{Si}$ doped $\mathrm{n}$ layer, a $25 \mathrm{~nm}$ Q1.2 spacer layer, three $6 \mathrm{~nm}$ thick compressively strained InAsP quantum wells (QWs) embedded in $15 \mathrm{~nm}$ Q1.2 barriers, a $25 \mathrm{~nm}$ Q1.2 spacer layer, a $135 \mathrm{~nm}$ thick $5 \times 10^{17}$ to $1 \times 10^{18} \mathrm{~cm}^{-3}$ Be doped InP layer, and the p-type contacts layers. Metal contacts have usually a poor resistivity on p-type InP, and in standard devices it is necessary to add a p-type InGaAs contact layer to improve the contact resistance. This InGaAs layer is highly absorbing around $1.55 \mu \mathrm{m}$ however. To overcome this issue, we replaced the $\mathrm{p}$-contact by a $\mathrm{p}+\mathrm{n}+$ tunnel junction and a $\mathrm{n}+$ contact layer. The top contact then constitutes of a $20 \mathrm{~nm}$ thick $\mathrm{p}+\left(2 \times 10^{19} \mathrm{~cm}^{-3}\right)$ InGaAsP emitting at $1.3 \mu \mathrm{m}(\mathrm{Q} 1.3)$ layer, a $20 \mathrm{~nm}$ thick $\mathrm{n}+$ $\left(1 \times 10^{19} \mathrm{~cm}^{-3}\right) \mathrm{Q} 1.3$ layer, and finally a $80 \mathrm{~nm} \mathrm{n}+\left(5 \times 10^{18}\right.$ to $1 \times 10^{18} \mathrm{~cm}^{-3}$ ) InP contact layer. This tunnel junction based contact can be used either as the bottom contact, as was the case in our earlier work [29] or as the top contact, which is the case here. When used as a bottom contact, the tunnel junction acts as an etch-stop layer during the fabrication of the bottom contact slab. However, in this configuration the tunnel junction is close to the waveguide below the microdisk and the optical losses due to the high p-doping level increases. Therefore, in our recent work we reversed the growth order and now the tunnel junction forms the top contact of the device, leading to lower optical confinement and hence lower optical losses in this layer.

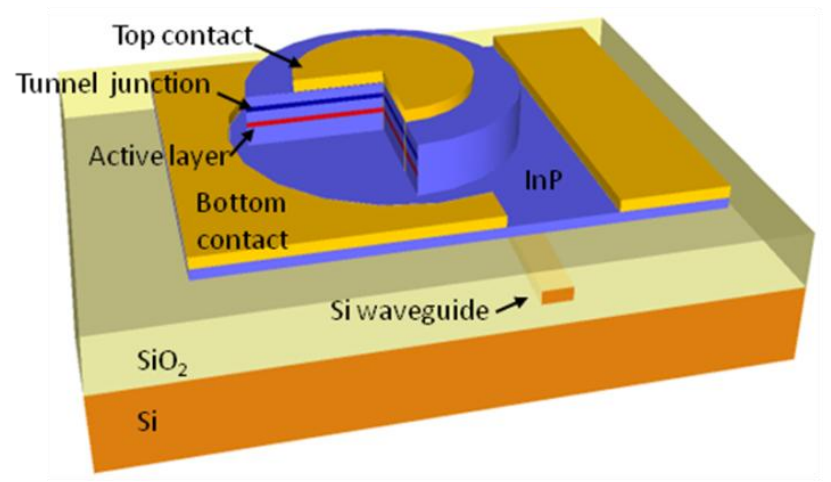

Fig. 1 Schematic representation of a microdisk laser heterogeneously integrated on SOI.

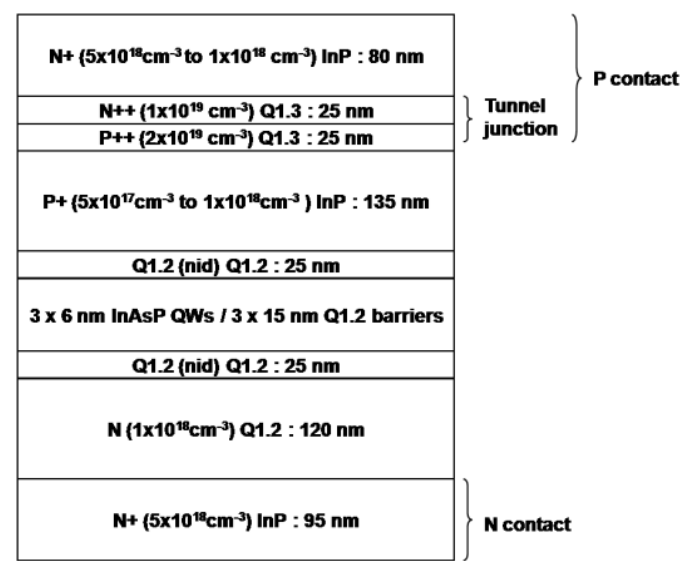

Table 1 Layer structure of improved microdisk design. Note: structure is grown upside down for compatibility with bonding process.

The new epitaxial structure has a total thickness of 580nm, which is significantly thinner than the previous structure (955 
$\mathrm{nm})$. This has a positive effect on the scattering loss and the coupling efficiency. It can be shown that, taking into account the large vertical confinement, the scattering loss, which is caused by the sidewall roughness of the microdisk, scales roughly linearly with the thickness of the device [32]. The coupling efficiency to the underlying waveguide also increases for a thinner structure, because there exists a larger exponential tail in the cladding, and thus also a larger overlap with the silicon waveguide.

In earlier devices, the low thermal conductivity of the dielectric layers surrounding the microdisk device, including the SOI box layer, the bonding layer and the passivation layers, resulted in severe self heating of the device [33]. Therefore, in the current design we implemented an improved thermal heat sink under the form of a thick layer of gold $(600 \mathrm{~nm})$ on the top contact of the microdisk. Although a thermal roll-over effect is still present, saturation of the output power now occurs around a drive current of $4 \mathrm{~mA}$ instead of $2 \mathrm{~mA}$ in previous devices.

For these devices continuous wave lasing at room temperature was observed for microdisks with a diameter of $7.5 \mu \mathrm{m}$ and $10 \mu \mathrm{m}$. Fiber grating couplers [34] were used to collect the light at one end of the silicon waveguide. The fiber coupled power for a $7.5 \mu \mathrm{m}$ diameter microdisk, is shown in

Fig. 2, together with the voltage-current response. A threshold current of $350 \mu \mathrm{A}$ and a maximum output power in the fiber of $38 \mu W$ was measured. The oscillations in the light output curve are due to reflections of the grating couplers, which are placed at both sides of the output waveguide. The abrupt change in the curve around $2.4 \mathrm{~mA}$ is related to a change in the lasing wavelength from $1554 \mathrm{~nm}$ to the next fundamental mode with a lower azimuthal mode number at $1584 \mathrm{~nm}$, due to self heating of the device. Since the output couplers are wavelength dependent, this at least partly explains the jump in the output power. The grating couplers have an efficiency of $21 \%$ at 1554 $\mathrm{nm}$ and $30 \%$ at $1584 \mathrm{~nm}$, which means the maximum power coupled into the waveguide is $120 \mu \mathrm{W}$. This is a considerable improvement compared to the previous generation. The optical spectrum when a $4 \mathrm{~mA}$ drive current is applied to the microdisk is shown in Fig. 3. The lasing wavelength is $1584 \mathrm{~nm}$ and the fundamental mode has a FSR of about $30 \mathrm{~nm}$. The side mode suppression ratio (SMSR) is $35 \mathrm{~dB}$. The power consumption of the device will be dominated by its static drive power, which is about $4 \mathrm{~mW}$ at $2 \mathrm{~mA}$ drive current. For a $10 \mathrm{GBit} / \mathrm{s}$ data stream this leads to a power consumption of $400 \mathrm{fJ} / \mathrm{bit}$, not including the dynamic part of the power consumption and the consumption in the driver circuits. Hence further improvements are needed to reach the 100fJ/bit target [35]. These could include single sided emission, reduced injection in centre of disk, smaller disk radius and improved processing.

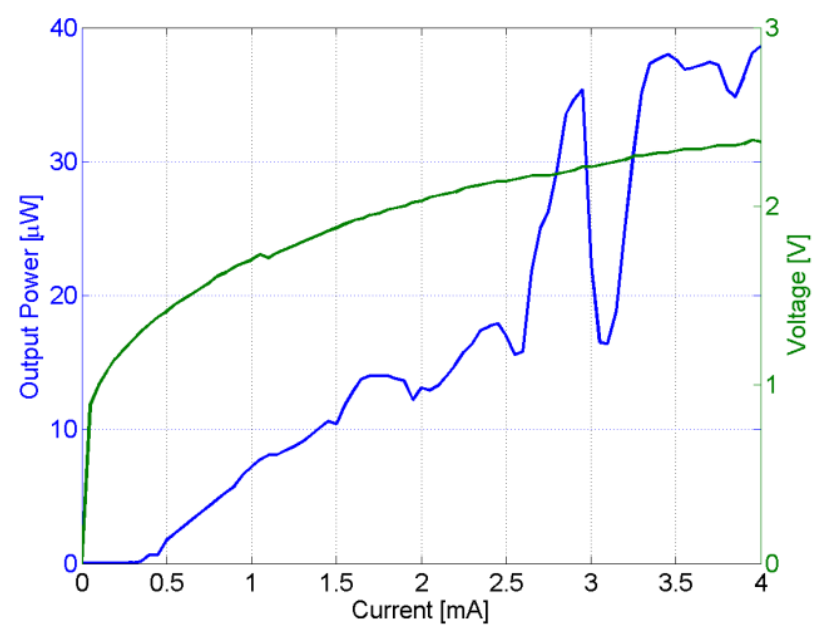

Fig. 2 LIV curves for a 7.5 $\mu \mathrm{m}$ diameter microdisk laser.

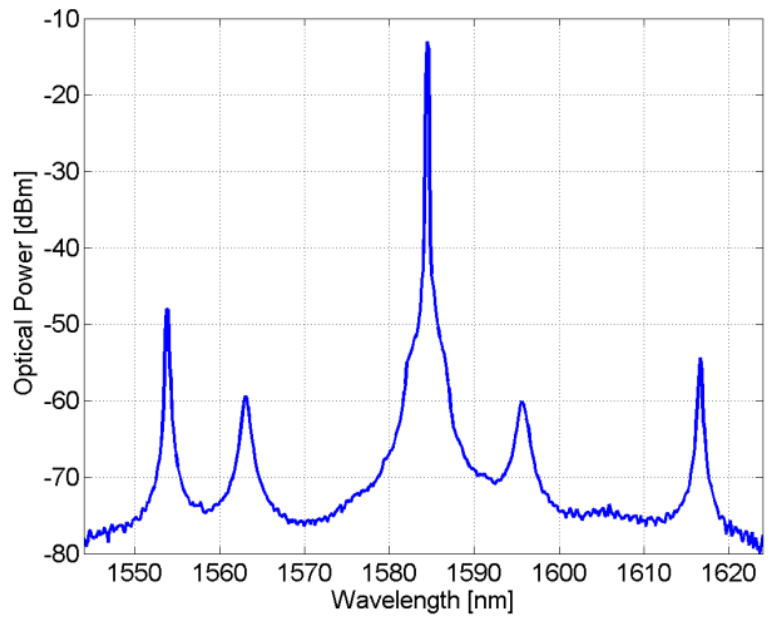

Fig. 3 Optical spectrum of a $7.5 \mu \mathrm{m}$ diameter microdisk.

\section{Multi-Wavelength Transmitter}

As discussed already in the introduction, WDM technology is essential for scaling up the communication capacity of a single link. Wavelength information can also be used to improve the functionality of an optical interconnect network, e.g. to control the routing of the communication signals towards the different cores within the network. For these applications a multi-wavelength laser (MWL) source, which can emit several wavelength signals, preferably at a predefined channel grid, is required. Such a laser can be implemented straightforwardly by cascading several microdisk lasers (MDLs) on a single silicon bus waveguide as shown in Fig. 4(a) [1]. Fig. 4b and c show the measured spectra of two 4-channel multi-wavelength lasers: one with $6 \mathrm{~nm}$ channel spacing, the other with $8 \mathrm{~nm}$ channel spacing. The bias currents of the MDLs have been adjusted individually, in order to achieve a uniform power for all channels. Due to the fabrication accuracy, the lasing wavelengths of identical micro-disks vary about $\pm 500 \mathrm{pm}$ within the same chip. Therefore, wavelength trimming, e.g. through the thermo-optical effect by implementing a local heater, is needed to align each of the lasing peaks to the 
designed channel grid. A possible implementation is discussed in the following section.
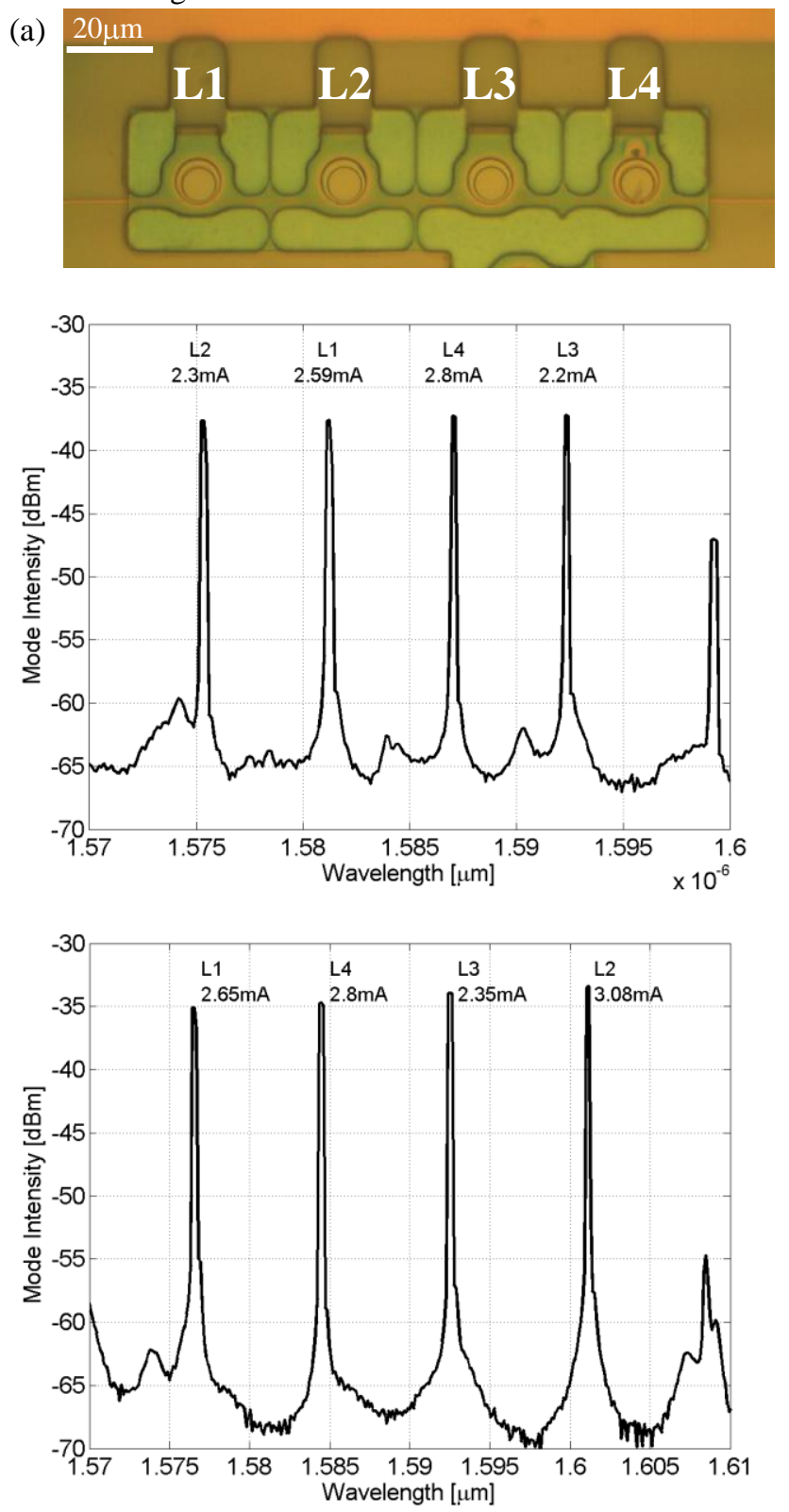

Fig. 4 (a) Fabricated multiwavelength laser before metallization, composed of four MDLs on a single silicon bus waveguide. (b-c) Spectra of multiwavelength lasers with $6 \mathrm{~nm}$ and $8 \mathrm{~nm}$ channel spacing. The bias current of each MDL and the laser number is indicated on the corresponding lasing peak [36].

\section{Dynamic response of microdisk laser}

The dynamic response of an MDL was tested by directly modulating the bias current, using first generation devices (having limited output power). Fig. 5 shows the response of a $7.5 \mu \mathrm{m}$ diameter MDL modulated with a periodic square-wave signal at $1.5 \mathrm{GHz}$. The low level of the drive signal was slightly above threshold, the high level was at the current giving the highest power. The extinction ratio of the output laser signal is approximately $10 \mathrm{~dB}$ after the amplification by an Er-doped fiber amplifier (EDFA). The rise and fall times are 110ps and $150 \mathrm{ps}$, respectively. Due to the low output power $(2 \mu \mathrm{W}$ coupled to fiber), eye diagrams nor bit error rate could not be measured reliably at this stage. It is believed that the data transmission performance will be improved using a MDL with the improved design as discussed above where over $30 \mu \mathrm{W}$ was measured in fiber.

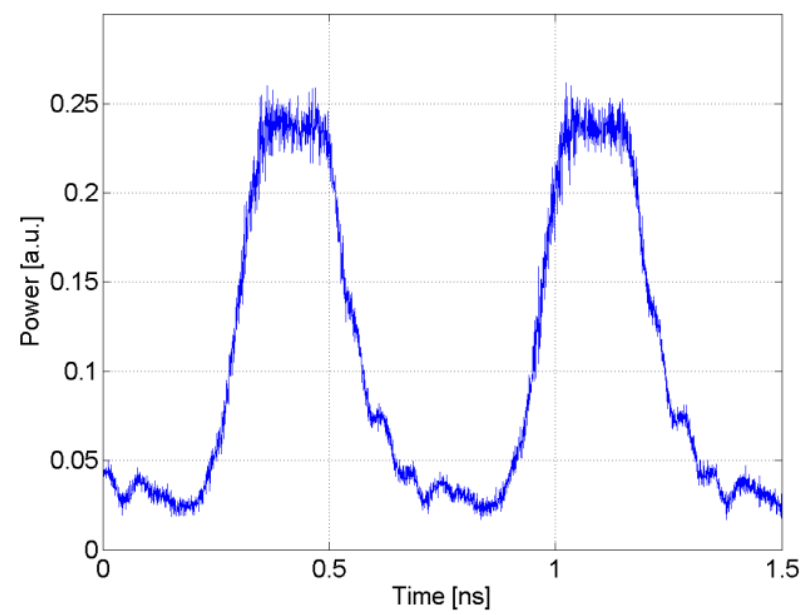

Fig. 5 Direct current modulation response of a $7.5 \mu \mathrm{m}$ diameter MDL with an electrical driving signal of a periodic square-wave at $1.5 \mathrm{GHz}$ [37].

\section{E. Tuning of microdisk laser wavelength}

For tuning the lasing wavelength, we propose a microdisk laser design with an integrated heater as shown in Fig. 6(a). The heater (which is fabricated in the same III-V layers also used for the disk) is a ring sitting around the microdisk cavity where the laser field is confined. Fig. 6b shows an SEM-picture of the structure immediately following the III-V etching step. The gap between the ring and the central disk is a critical parameter, and should both be small enough to allow for efficient heating and large enough to avoid leakage of the cavity whispering gallery mode to the heater. Simulation suggests that $1.0 \mu \mathrm{m}$ $2.0 \mu \mathrm{m}$ is an optimal value. Fig. 6(c) shows the calculated temperature field distribution when the diameter of the disk is $7.5 \mu \mathrm{m}$ and the gap is $1.6 \mu \mathrm{m}$. The heating efficiency determined at the edge of the central disk is about $3.4 \mathrm{~K} / \mathrm{mW}$, corresponding to a tuning rate of $0.34 \mathrm{~nm} / \mathrm{mW}$.

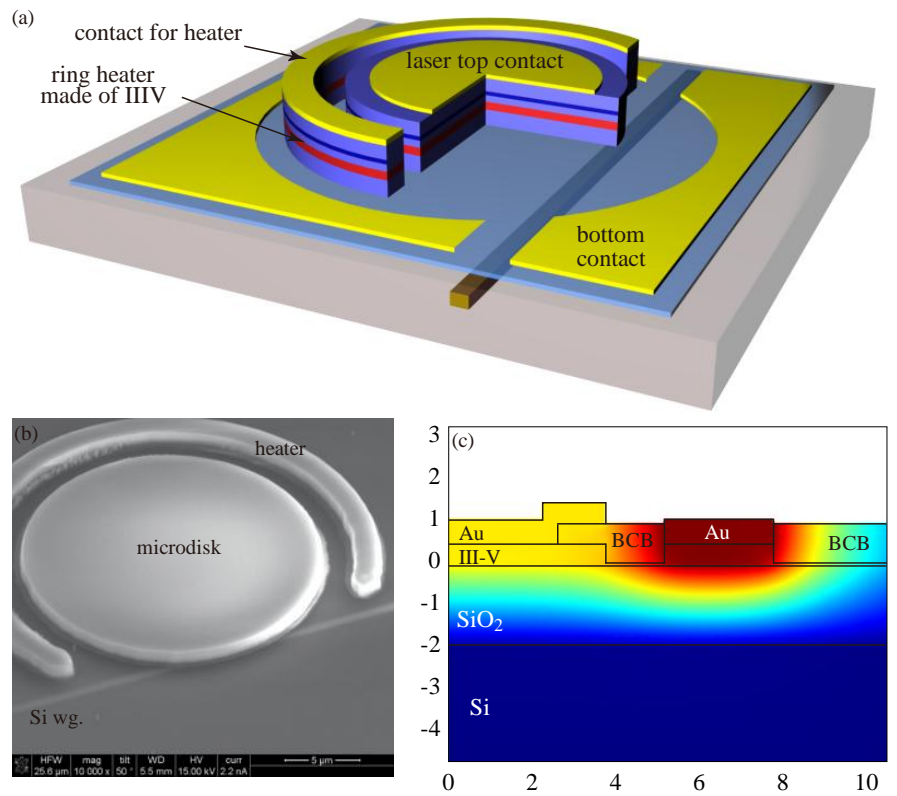


Fig. 6 (a) sketch of the microdisk laser with an integrated ring heater consisting of a forward biased III-V pin-junction. (b) Scanning electron microscope picture of the microdisk cavity and ring heater. (c) Temperature distribution in the proposed device.

Fig. 7 shows the lasing spectra from the microdisk laser with different electrical powers applied to the integrated heater. The laser driving current was fixed at $1.5 \mathrm{~mA}$. The tuning rate was measured to be $0.31 \mathrm{~nm} / \mathrm{mW}$, close to the simulated result. A $2.2 \mathrm{~nm}$ shift without severe impact on the lasing power could be demonstrated. Larger tuning is possible by applying a higher current to the heater, but comes at the expense of decreasing laser output power.

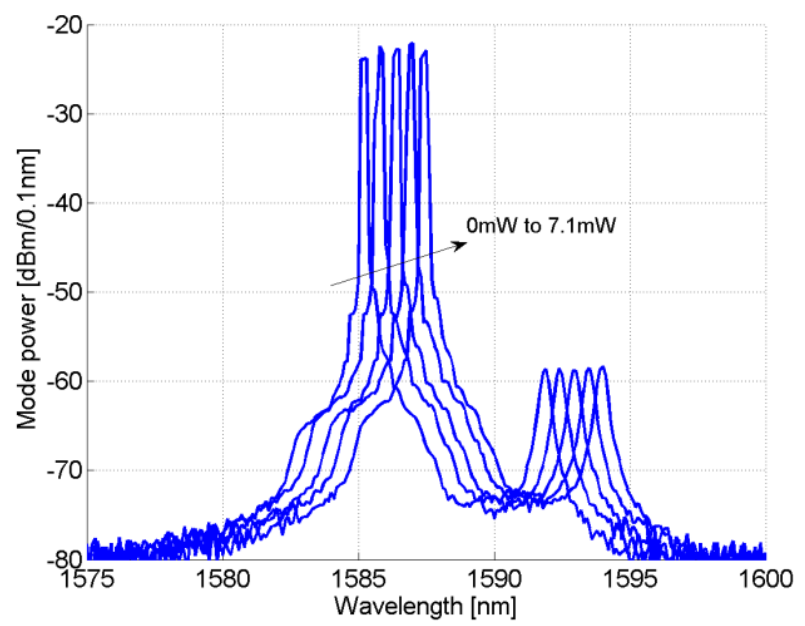

Fig. 7 Tuning of the microdisk laser wavelength. Along the arrow direction, the powers applied to the integrated heater are $0 \mathrm{~mW}, 1.6 \mathrm{~mW}, 3.5 \mathrm{~mW}, 5.3 \mathrm{~mW}$, and $7.1 \mathrm{~mW}$, respectively.

\section{F. Design of microdisk with external cavity}

The modes in the standard microdisk laser can be labeled by a set of numbers $(l, m, n)$ denoting the radial $(l)$, azimuthal $(m)$ and vertical $(n)$ mode number [38]. By careful design of the metal top contact and the position of the gain layer the modes $(0, m, 0)$ will have a considerable lower intrinsic loss rate $\tau_{0}^{-1}$ compared to the higher order modes with non zero $l$ and $n$ numbers and will have a much lower threshold gain [31]. However, there is no clear mechanism to select the azimuthal mode number $m$. For microdisks with radius larger than $2 \mu \mathrm{m}$, the free spectral range (FSR) will typically be smaller than the gain bandwidth and lasing in multiple orders may occur. In addition, both clock wise and counter clock wise lasing may occur, which may be undesirable in practical applications or result in decreased usable output power. To resolve these issues, we propose a new configuration as shown in Fig. 8. At one side the microdisk is coupled to an external cavity, in the silicon waveguide layer. At the other side, the two outputs are coupled together using e.g. a multi-mode interference coupler (MMI).

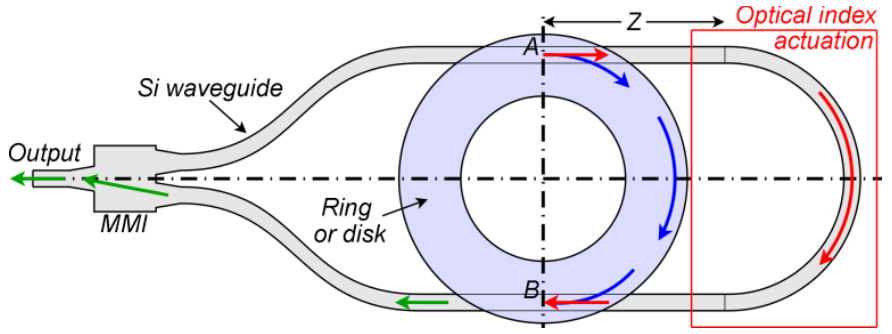

Fig. 8 Microdisk coupled to an outer loop, with a single optical output.

Then, using coupled mode theory [39], we can demonstrate that the phase difference $\Delta \varphi_{A B}$ between the two possible optical paths going from $\mathrm{A}$ to $\mathrm{B}$ both controls the resonance frequency $\omega$ and the quality factor $Q$ of the lasing mode:

$\left\{\begin{array}{c}\omega=\omega_{0}-\frac{1}{2} A_{g} \tau_{c}^{-1} \sin \left(\Delta \varphi_{A B}\right) \\ Q=\frac{\omega}{\tau_{0}^{-1}+\tau_{c}^{-1}\left(1+A_{g} \cos \left(\Delta \varphi_{A B}\right)\right)}\end{array}\right.$

Hereby $A_{g}$ is defined such that $\left(1-A_{g}\right)$ represents the relative amplitude attenuation for the mode propagating in the passive waveguide path connecting points $A$ and $B . \omega_{0}$ is the resonance frequency of the cavity without the external racetrack. The coupling rate between the disk and a single waveguide is given by $\tau_{c}$ and $\tau_{0}$ determines the intrinsic cavity lifetime. By controlling the relative phase difference $\Delta \varphi_{A B}$ and for low losses in the waveguide we now can:

- Vary the quality factor $Q$ between the values for a standard microdisk coupled to two waveguides without feedback ( $\approx \frac{1}{2} Q_{c}=\frac{1}{2} \tau_{c} \omega$ for constructive interference) and the intrinsic quality factor $Q_{0}=\tau_{0} \omega$ (for destructive interference).

- Adjust the resonance frequency $\omega$ by $\pm \frac{\tau_{c}^{-1}}{2}$.

Fig. 9 shows the evolution of the $\mathrm{Q}$ factor as a function of the external waveguide length $Z$ (which controls the phase difference $\Delta \varphi_{A B}$ ) for 5 modes with increasing azimuthal mode number $n$ of a $4 \mu \mathrm{m}$ radius microdisk. Depending on the external waveguide length $\mathrm{Z}$, we denote a different overall behavior. For specific sizes of the external loop (e.g. a few $\mu \mathrm{m}$ in Fig. 9a), the modes are regularly distributed. Then, by controlling the refractive index of the material, e.g. thermally or through carrier injection, we can fine tune the resonant wavelength, e.g. to compensate small fabrication variations, while still maintaining a high contrast in quality factor between the different azimuthal modes and hence ensuring single mode operation. In the case of Fig. $9 \mathrm{~b}$ the odd modes and even modes each almost share the same phase condition. The frequency separation between two modes with high Q-factor is doubled, resulting in a doubling of the practical FSR and hence higher SMSR. In Fig. 9c all modes almost share the same phase condition. Then a slight index change in the external waveguide leads to a mode jump. In this particular example, we can switch from $\lambda=1.643 \mu \mathrm{m}$ to $\lambda=1.490 \mu \mathrm{m}$ with only a few $\%$ variation in the optical paths (i.e. $Z$ from 14.44 to $14.47 \mu \mathrm{m}$ in terms of waveguide lengths). If the FSR of the microdisk corresponds to the channel spacing in the communication system, we can now hop to the different channels by using only a very small refractive index change. 

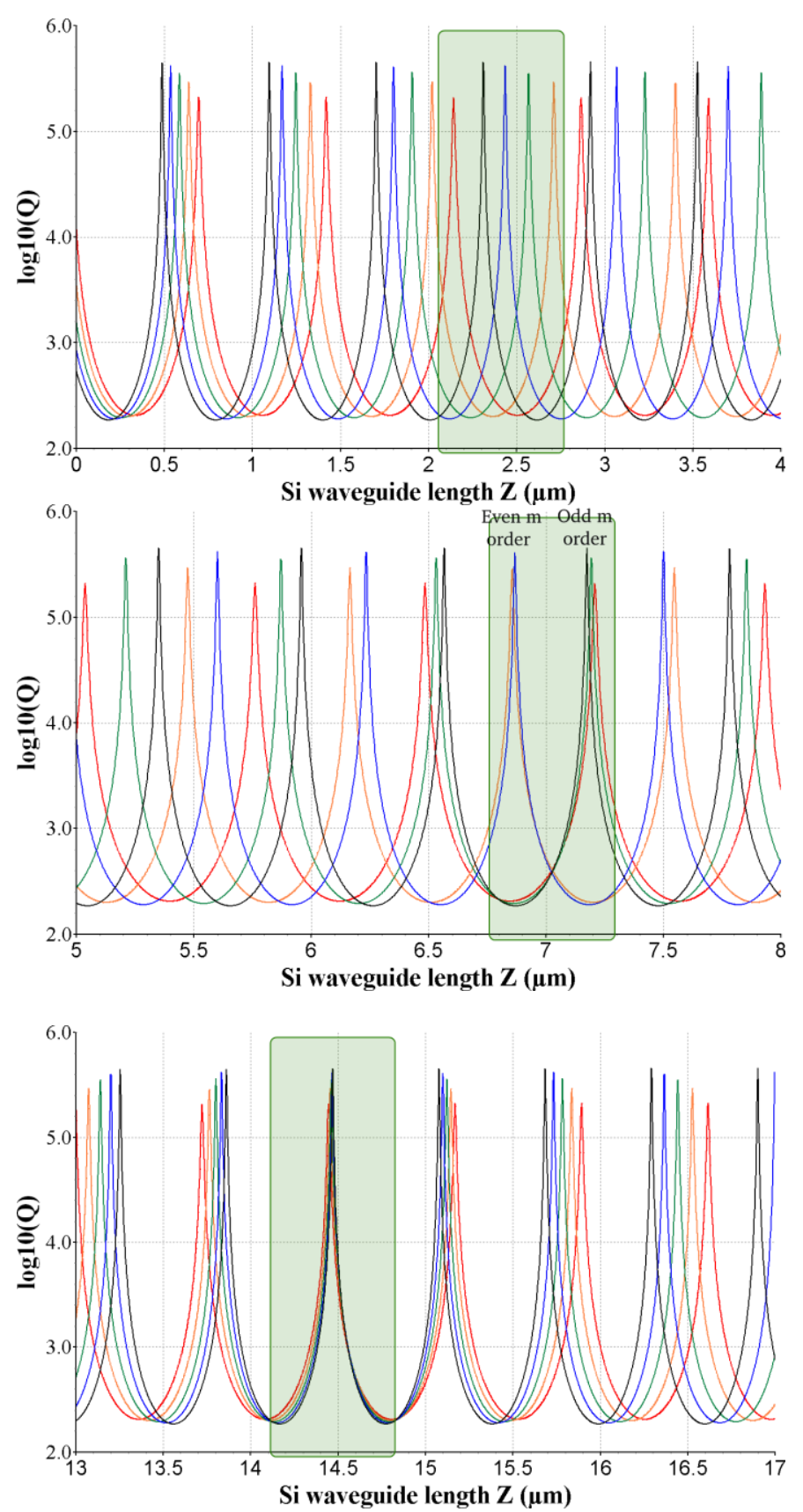

Fig. 9 Q factor variation as function of external loop length for resonant modes of a $4 \mu \mathrm{m}$ radius and $0.55 \mu \mathrm{m}$ thick microdisk coupled to a $200 \times 500 \mathrm{~nm} \mathrm{Si}$ waveguide. Differently coloured curves corresponds to the $\mathrm{TE}(0,31,0)=1645 \mathrm{~nm}$ up to the $\mathrm{TE}(0,35,0)=1490 \mathrm{~nm}$ resonances of the resonator. The minimum value of $\mathrm{Q}$ can be arbitrary low and is determined by the coupling rate.

\section{ADVANCED FUNCTIONALITY OF MICRODISK LASER}

\section{A. Microdisk EO-switch}

Also low power optical switching is an essential network functionality. Here we demonstrate that a microdisk laser coupled to two bus waveguides (Fig. 10a) can be used to switch an input digital signal between two outputs. The switching is based on the plasma dispersion effect, which induces a shift in the resonance wavelength when injecting current in the microdisk cavity. The experiments were carried out using a $10 \mu m$ diameter microdisk. Forward biasing the microdisk blue shifts the resonance as shown in Fig. 10. The best working point for switching was found to be $55 \mu \mathrm{A}(0.9 \mathrm{~V})$. The bandwidth of the switch at resonance was $0.7 \mathrm{~nm}$, in principle allowing to switch $10 \mathrm{~GB} / \mathrm{s}$ and even $40 \mathrm{~GB} / \mathrm{s}$ data signals. In the current device, the drop port loss was $10 \mathrm{~dB}$, which is probably due to a slight unbalance between the coupling coefficients to the bus waveguides related to a misalignment in the microdisk fabrication. To demonstrate the dynamic response of the proposed switch, we used a $10 \mathrm{GHz}$ periodically modulated optical signal (center wavelength at $1610.19 \mathrm{~nm}$ ) as the input beam, and the bias of the microdisk was swept from $-1.1 \mathrm{~V}$ (negligible current) to $+0.9 \mathrm{~V}(55 \mu \mathrm{A})$. The results are shown in Fig. 11. Switching from drop port to through port takes about 70 ps. However, in the opposite direction the switching time is about $1.2 \mathrm{~ns}$ due to the relaxation oscillation in the carrier-injection transient. The dynamic extinction ratio is about $15 \mathrm{~dB}$, similar to that of the static case as shown in Fig. 10 .

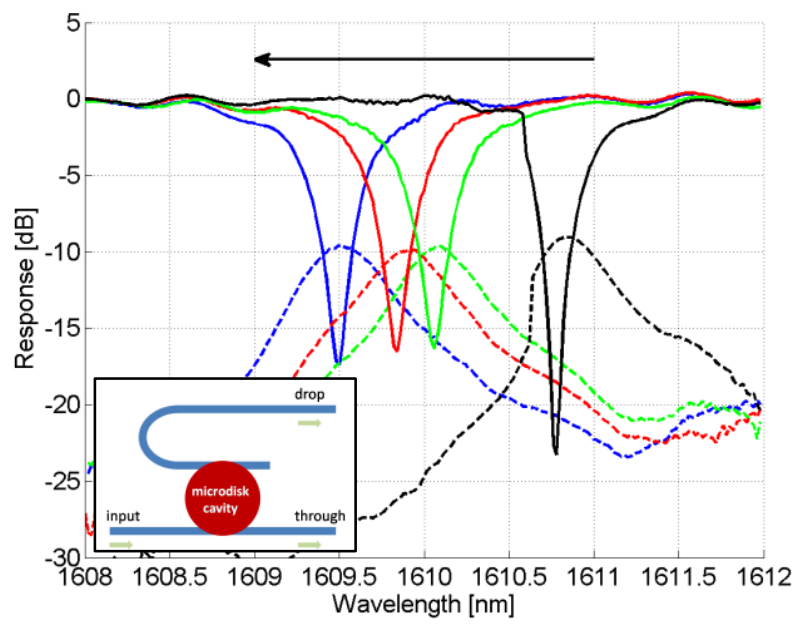

Fig. 10 Schematic layout of the proposed switch (inset) and the influence of the injection current on the through (solid) and drop (dashed) port transmission characteristics of the microdisk cavity (curves for $0 \mathrm{uA}, 55 \mathrm{uA}(0.9 \mathrm{~V}), 100 \mathrm{uA}$ $(1 \mathrm{~V})$ and $200 \mathrm{uA}(1.1 \mathrm{~V}))$

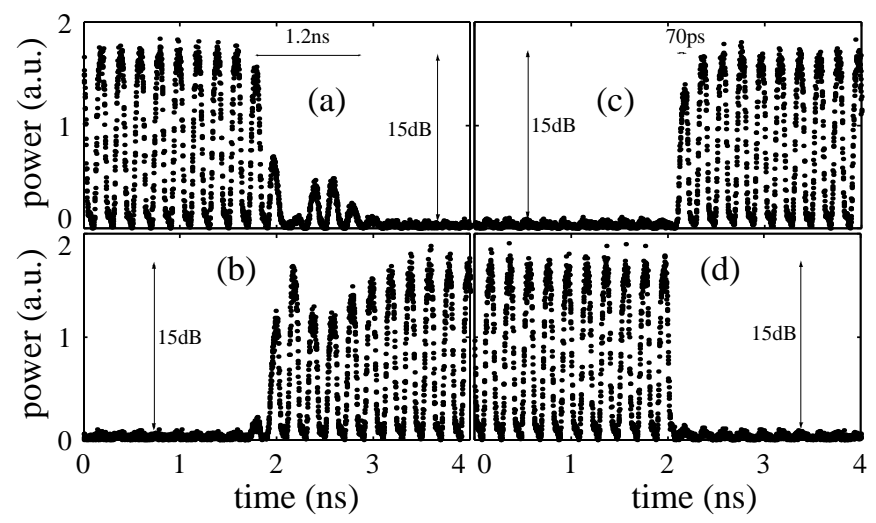

Fig. 11 Switching characteristics of the microdisk electro-optical switch. (a) and (c) are the off and on transients at the through port, respectively; (b) and (d) are the on and off transients at the drop port, respectively 


\section{B. All-optical wavelength conversion}

To improve the scalability of wavelength routed networks, all-optical wavelength conversion is an important function [15]. In [40] we demonstrated for the first time that the microdisk laser can be used as an ultra-low power wavelength convertor. Static wavelength conversion with a control power of $6.4 \mu \mathrm{W}$ and dynamic conversion using a $5 \mathrm{~GB} / \mathrm{s}$ non-return-to-zero bit sequence was demonstrated. Recently, we improved these results further and demonstrated up to $10 \mathrm{~GB} / \mathrm{s}$ wavelength conversion [41] (using first generation microdisk lasers). The experimental setup is shown in Fig. 12. The pump signal was tuned to a non-dominant lasing mode of the microdisk laser. The converted signal (at the dominant laser mode) is extracted using a circulator and is inverted with respect to the input signal. In this second experiment we also added a seed laser (tuned to the dominant laser mode), which supports the recovery of the laser signal.

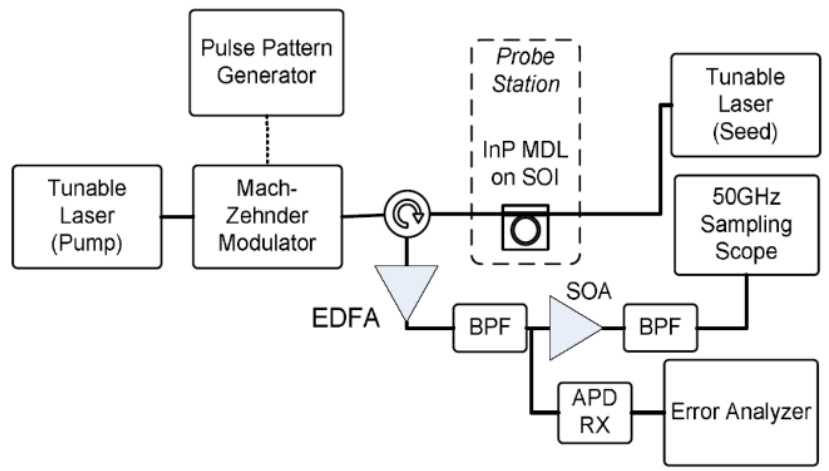

Fig. 12 Experimental setup for micro-disk based all optical wavelength conversion (from [41]).

Using this setup we measured the bit-error-rate at $2.5 \mathrm{~GB} / \mathrm{s}$. Due to the weak output power the bit-error-rate at $10 \mathrm{~GB} / \mathrm{s}$ could not be determined but clearly inverted signals were obtained using a sampling scope.

\section{All-optical flip-flops}

It has been shown before that ring or disk lasers can be used to implement all-optical flip-flops [42], with switching taking place between two unidirectional (clockwise-CW and counterclockwise-CCW) lasing states. Theoretical work has revealed however that such unidirectional operation is only possible if the coupling between the $\mathrm{CW}$ and the $\mathrm{CCW}$ lasing modes is sufficiently small and if the photon density in the lasing mode is sufficiently large [43].

In the heterogeneously integrated microdisk lasers described above, we can obtain high power density in the whispering gallery mode owing to the high index contrast of the InP membranes. Due to the improved heat sinking obtained through the thick top contact, we can also drive the microdisk lasers up to relatively high currents and high power levels.

Coupling between CW and CCW modes is mainly caused by the scattering at the disk surface and by the reflections in the silicon waveguides at the grating couplers. This coupling was substantially reduced when using the optimised lithography and etching processes discussed in section II, resulting in minimum sidewall surface roughgness. In addition, we have employed a relatively thick $(250 \mathrm{~nm})$ DVS-BCB bonding layer to reduce the coupling between disk and straight SOI waveguides and thus to reduce the influence of the reflections at the grating couplers.

A typical power vs. current characteristic, measured at both ends of the silicon waveguide, is shown in Fig. 13. Unidirectional operation is obtained from $1.7 \mathrm{~mA}$ onwards. Due to the single mode operation of the laser, we did not observe any switching of the unidirectional operation when changing the bias current.

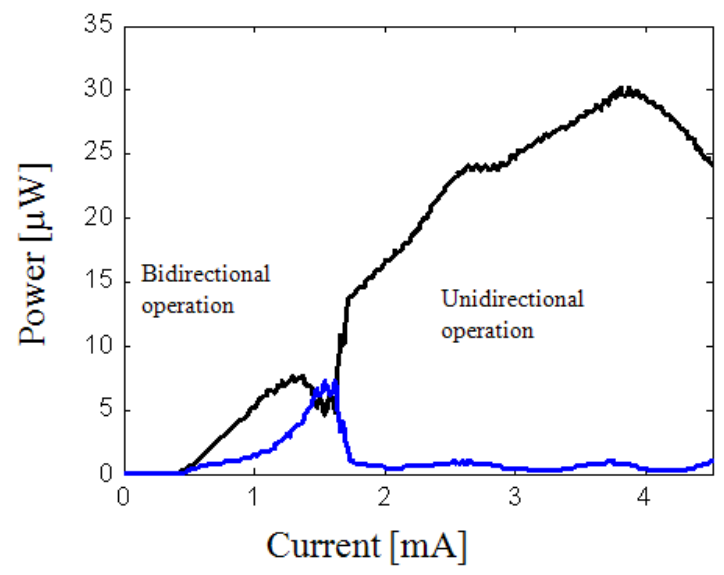

Fig. 13 Typical output power versus current characteristic of a microdisk laser for AOFF operation, measured at both ends of the SOI waveguide.

Switching between $\mathrm{CW}$ and $\mathrm{CCW}$ modes in disk and ring lasers is achieved through injection locking. Optical pulses at the laser wavelength injected at the left, resp. right hand side lock the CCW, resp. CW laser mode and cause switching off of the $\mathrm{CW}$, resp. CCW mode. We performed switching experiments using $100 \mathrm{ps}$ long set and reset pulses, which were alternatively injected to the left and the right grating couplers and biased the microdisk laser at $3.5 \mathrm{~mA}$ (roughly twice the threshold for unidirectional operation). The output power measured from the right grating coupler, showing switching on and off of the $\mathrm{CW}$ mode, is shown in Fig. 14. Due to the design of the device (with only one straight waveguide coupled to the disk), it was not possible to separate the switching pulses and the laser output. Switching was obtaind in less than 100 ps using pulses with an energy of only $1.8 \mathrm{fJ}$. For more details we refer to [44].

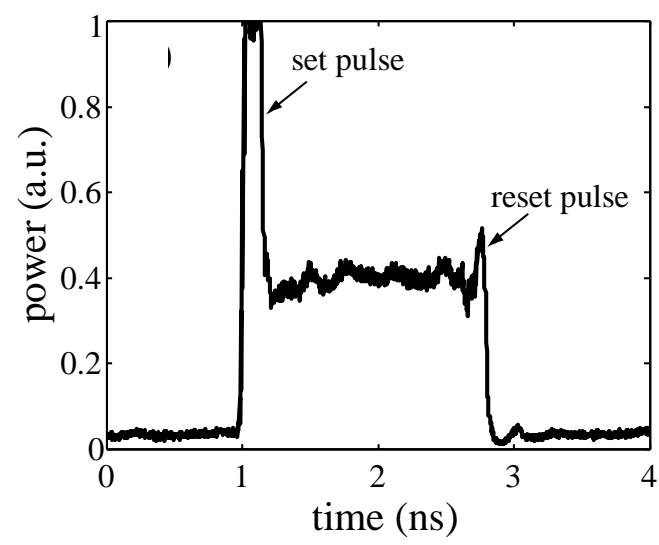


Fig. 14 Switching on and off of the microdisk laser, at a bias current of $3.5 \mathrm{~mA}$ and using 100ps long pulses.

\section{Optical gating}

Optical gating can be implemented using the microdisk lasers below their lasing threshold current or even without or with negative bias. In both cases it is based on carrier density changes induced by the data, which cause changes in the microdisk resonances that are in turn affecting the transmission of clock pulses.

We performed gating experiments using microdisk lasers with $10 \mu \mathrm{m}$ radius and the improved design described in section IIB. We applied a bias varying from 0 to -2 Volt and used a $\mathrm{CW}$ probe beam in combination with a pump beam consisting of $10 \mathrm{GHz}$ pulses. Fig. 15 shows the transmission at the through port versus wavelength for bias voltages of $-2,-1$ and $0 \mathrm{~V}$ and for a probe beam of $0.5 \mathrm{~mW}$, which fully saturates the absorption. Rise and fall times together were 50ps.

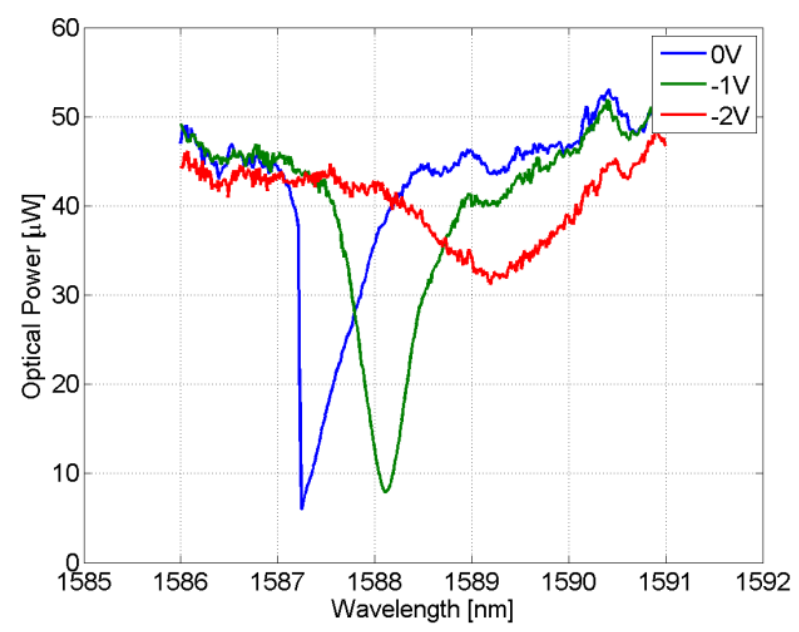

Fig. 15 Transmission at the through port of a negatively or zero biased microdisk laser vs. wavelength of the probe beam.

\section{PASSIVE OPTICAL ROUTING DEVICES}

\section{A. Fabrication accuracy of silicon nanophotonic circuits}

One of the major drawbacks of high index contrast waveguide platforms is the very limited tolerance for manufacturing imperfections. Slight deviations in device dimensions (height and width) can cause considerable shift in the spectral response of the filters used for routing and selecting wavelength channels. In complex circuits such as those proposed for on-chip optical networks [11-13], which consists of a concatenation of multiple wavelength dependent devices, this leads to high losses or even errors in the routing process. We investigated in detail the performance of the currently available process, based on 193nm DUV lithography and dry etching [45]. We found that the variations manifest themselves at two different levels: variations over a single die and variations over a wafer. At both levels the photolithography and the dry etching process are the two main causes for linewidth variations during pattern definition. Next to these, waveguide thickness variations originating from the base wafer (SOI) may further induce resonant wavelength variations. By careful process design the non-uniformity of the devices can be improved considerably. We have demonstrated that a device non-uniformity of $<0.6 \mathrm{~nm}$ within a die and $<2 \mathrm{~nm}$ within a wafer can be achieved [46, 47]. Further enhancement requires improvement of the circuit design however. To illustrate this, we investigated a design containing 4 ring resonators separated by $1.5 \mathrm{~mm}$. In the neighborhood of the ring resonators the overall device density (density of patterns around the ring resonators under study) changes abruptly. The design was set up in such a way that this change was relatively small in the neighborhood of the first ring and then gradually increased towards the fourth ring. We then measured the uniformity of the resonance wavelength of the four rings from 11 chips selected from a single $200 \mathrm{~mm}$ wafer.

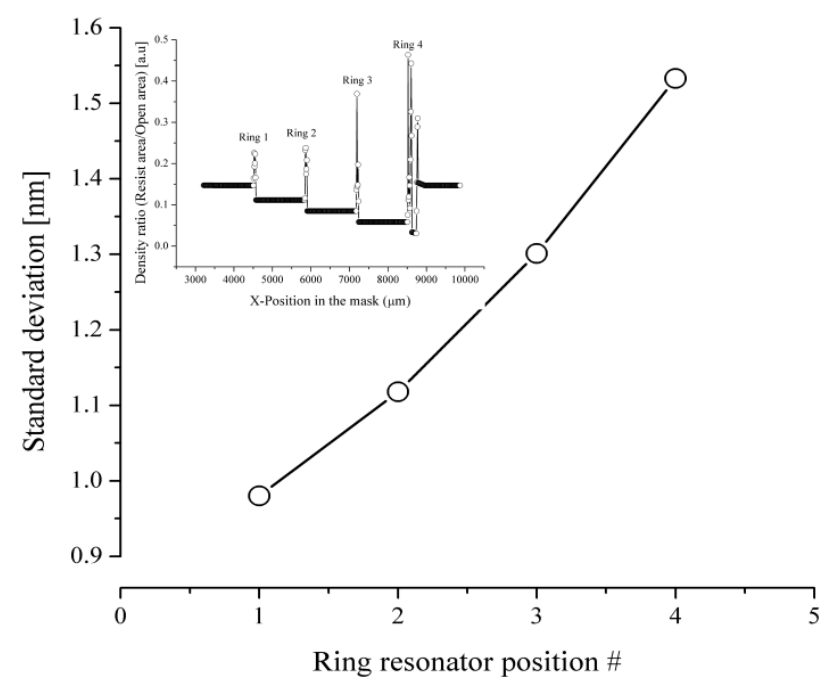

Fig. 16 Non-uniformity of four ring resonators with different local pattern density over a $200 \mathrm{~mm}$ wafer. The inset shows the local pattern density.

To avoid any environmental disturbances, the circuits were characterized in a cleanroom environment. From the results in Fig. 16 it can be clearly seen that abrupt changes in the device density (ring 4) create considerable device non-uniformity $(\sigma=$ $1.55 \mathrm{~nm}$ ), while ring 1 , which had the lowest variation has also lower non-uniformity $(\sigma=0.98 \mathrm{~nm})$. Thus, designing the circuits in such a way that the device density is kept as uniform as possible is important in achieving the optimum uniformity.

As a further test for the high resolution fabrication process we designed higher order ring resonator based filters. Both coupled cavity filters (CCF) and all-pass filters (APF) were implemented, using race track resonators as the base element. In case of the $\mathrm{CCF}$ from 10 up to 30 identical ring resonators were side coupled to each other and the coupling gap between the resonators was tuned to reduce the pass-band ripple [48]. Fig. 17 depicts the spectral response of a $10^{\text {th }}$ order CCF filter. Despite the variation in the coupling rate a pass-band ripple of approximately $2 \mathrm{~dB}$ is observed, which we believe to be related to mask discretization errors (a $5 \mathrm{~nm}$ grid was used). The increased passband width of these filters (1.8nm FWHM) can be used to compensate for variations in the laser wavelengths or filter resonances. The APF configuration is implemented by cascading identical race-track ring resonators to a common bus waveguide (see inset Fig. 18). The spectral response of two identical APFs with 200 rings each is shown in Fig. 18. The 
response of the two filters is very similar, which demonstrates the uniformity of the fabrication process.

In literature, it was suggested that these devices can be used as a compact optical buffer [49]. Therefore we also measured the optical delay through higher order APFs and CCFs with up to 200 resonators, for which we found a delay of $6.0 \mathrm{ps} /$ ring and $1.2 \mathrm{ps} /$ ring respectively. Even though the APF configuration yields substantial higher delays, the insertion loss at resonance is very high making them at this moment impractical for implementing an optical buffer.

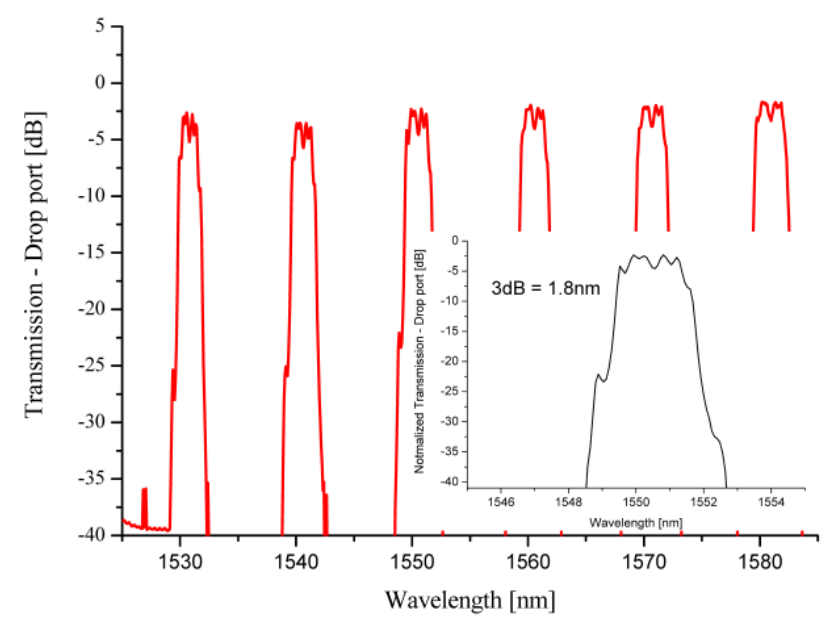

Fig. 17 Spectral response of a $10^{\text {th }}$ order CCF, inset shows details of a single wavelength band.

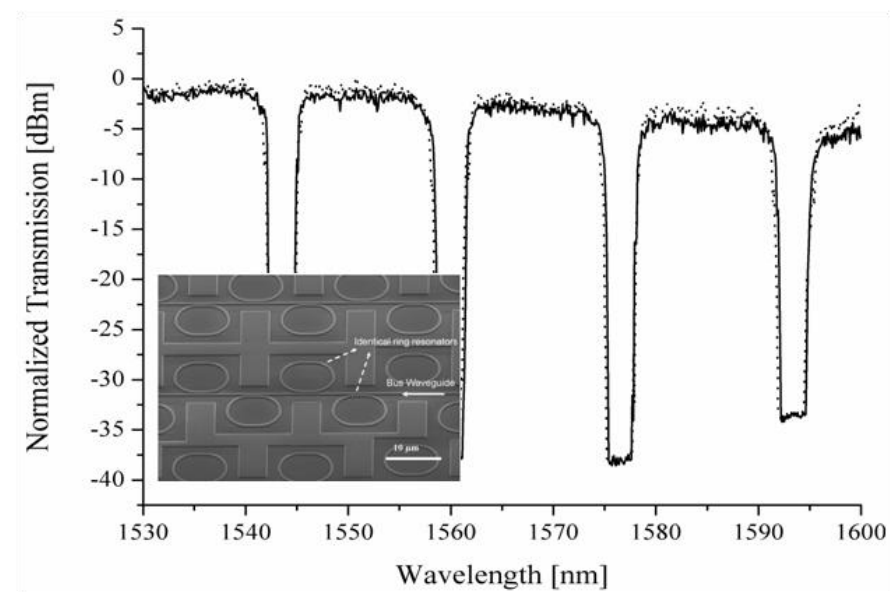

Fig. 18 Spectral response of two APF devices with 200 identical race-track ring resonators coupled to a common bus waveguide. Inset shows SEM picture of the APF.

\section{B. Thermal tuning of silicon nanophotonic circuit}

Despite the progress in fabrication technology, in many cases further fine tuning of the wavelength selective devices is required. Here we present thermo-optic tuning of microring based filters. The filters and the heaters discussed in this section where fabricated using $248 \mathrm{~nm}$ DUV lithography. Following definition of the waveguides, they were covered by $\mathrm{SiO}_{2}$ grown at low temperature and further planarized by chemical mechanical polishing. A 550nm thick layer remained on top of the microring resonators, which is a compromise between minimizing the optical loss and optimizing the heat transfer. The heaters consist of a $100 \mathrm{~nm}$ thick titanium layer (sheet resistance of $6 \Omega / \square$ ), which is compatible with the fabrication constraints of the CMOS pilot line. The inset of Fig. 19 shows a top view of the fabricated device.

Taking into account a thermo-optic effect $\mathrm{dn} / \mathrm{dT}=+1.8 \times 10^{-4} \mathrm{~K}^{-1}$ for silicon, we expect a spectral shift of $0.1 \mathrm{~nm} /{ }^{\circ} \mathrm{C}$ for the silicon ring resonators. We simulated the proposed heater using a finite element method and found a thermal resistance of $4600^{\circ} \mathrm{C} / \mathrm{W}$ for the $2 \mu \mathrm{m}$ silica layer between the titanium heater and the silicon substrate, in which the silicon waveguide is encapsulated. We also derived that $10 \mathrm{~mW}$ heat dissipation leads to an increase of the heater temperature by $46^{\circ} \mathrm{C}$. The temperature of the silicon ring resonator, which is $550 \mathrm{~nm}$ below, increases by up to $35^{\circ} \mathrm{C}$. Therefore, the theoretically expected spectral shift is $0.35 \mathrm{~nm} / \mathrm{mW}$.

The ring resonators of the 8-channel add-drop were designed to have either a free spectral range (FSR) of $32 \mathrm{~nm}$ or $23 \mathrm{~nm}$. Within the same circuit, slight changes in the microring radii ensure a channel spacing $\Delta \lambda$ between adjacent channels of $4 \mathrm{~nm}$ $(\mathrm{FSR}=32 \mathrm{~nm})$ or $3 \mathrm{~nm}(\mathrm{FSR}=23 \mathrm{~nm})$ to cover the whole FSR. Fig. 19 shows the experimentally measured FSR $(29 \mathrm{~nm}$ and $22 \mathrm{~nm}$ resp.) and channel spacing ( $3.3 \mathrm{~nm}$ and $2.8 \mathrm{~nm}$ resp.). The slight deviation between simulated and measured values is related to an underestimation of the waveguide group index.

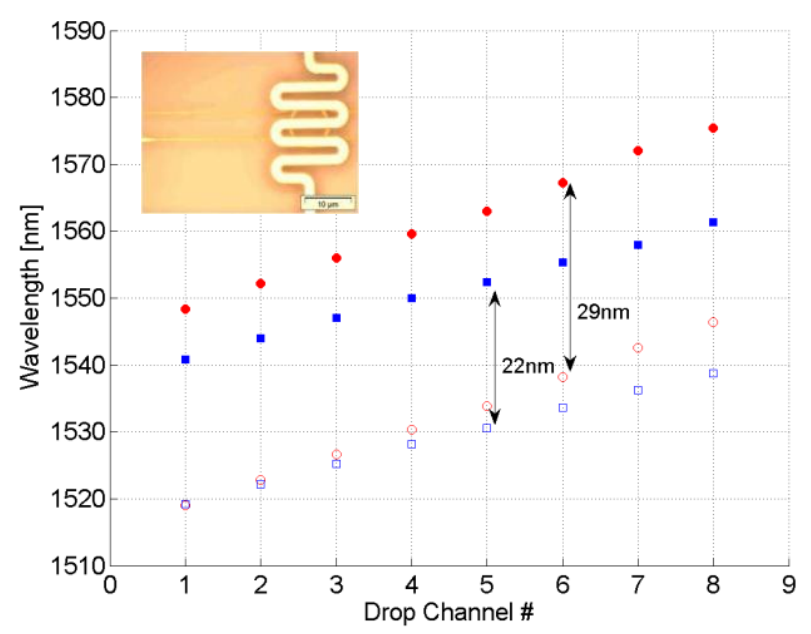

Fig. 19 Experimentally measured channel wavelengths for two 8-channel add-drop multiplexers with resp. $29 \mathrm{~nm}$ (circles) and $22 \mathrm{~nm}$ (squares) FSR. The inset shows a microscope image of the fabricated heater with underlying ring resonator.

Tuning of the microring resonant wavelength by dissipating power in the heater is shown in Fig. 20. A linear red shift of $0.3 \mathrm{~nm} / \mathrm{mW}$ is observed, which correspond to an efficiency of $26 \mu \mathrm{W} / \mathrm{GHz}$, comparable with the best values reported in literature $[50,51]$. The maximum power that can be dissipated in the heaters is $40 \mathrm{~mW}$, so tuning over the full channel spacing is possible without any problem. We confirmed that there is no thermal crosstalk between two adjacent ring resonators, which were spaced $200 \mu \mathrm{m}$ apart (center to center). 


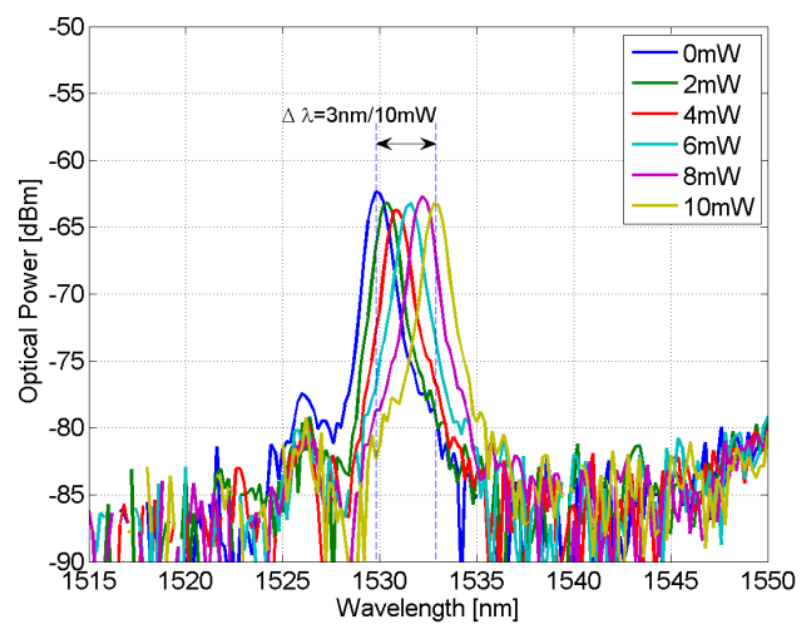

Fig. 20 Tuning of spectral response for increasing power consumption.

\section{WAVELENGTH SELECTIVE DETECTORS}

Wavelength selective detection is necessary for the aforementioned optical interconnect involving WDM and wavelength routing networks. We recently demonstrated a compact resonant metal-semiconductor-metal photodetector by integrating a short III-V absorbing mesa directly onto an SOI ring resonator [52]. Here, we further expand the functionality of this device by integrating a local heater for tuning the detection wavelength. Fig. 21 shows some pictures of a fabricated device. The InGaAs absorption layer was first bonded on top of the SOI circuits by using DVS-BCB polymer (see [53] for details on layer structure). Then the mesa, with a width of $3 \mu \mathrm{m}$, was defined by optical lithography and wet chemical etching. In the next step, a DVS-BCB overcladding layer was spun onto the whole chip for isolating the SOI circuits from the metal wires. A via was opened on top of the III-V mesa. A 100nm thick Ti layer (heater) was subsequently deposited on some part of the SOI ring. Finally, a Ti/Au metal layer was deposited to form the Schottky contacts and the wiring.
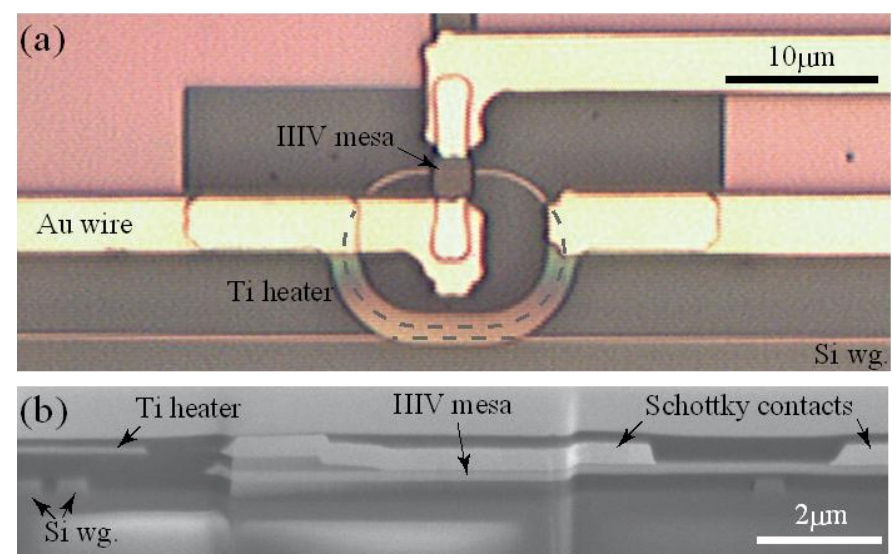

Fig. 21 (a) top view and (b) cross-sectional view of a fully fabricated resonant detector.

Fig. 22(a) shows the optical transmission and the detected current of a fabricated device at a bias voltage of 7V. Obviously, the detected current shows a wavelength selective response. At the resonant wavelength, the detector responsivity reaches $0.47 \mathrm{~mA} / \mathrm{mW}$. Off resonance, the detected current drops by about $10 \mathrm{~dB}$. In the transmission spectrum, resonant dips can also be observed, but the extinction ratio is only about 6-10dB. Note that the responsivity and extinction ratio shown here do not yet reach the theoretical optimum. By carefully designing the coupling ratios from the ring cavity to the SOI bus waveguide and to the III-V absorption mesa, up to $1 \mathrm{~A} / \mathrm{W}$ has been achieved [52]. The present device also exhibits a shoulder voltage of $3 \mathrm{~V}$, and a dark current of $0.1 \mathrm{nA}$ up to $10 \mathrm{~V}$ bias. By sending a current through the integrated Ti heater, the resonant wavelength of the present device can be tuned, as shown in Fig. $22 \mathrm{~b}$ ). A maximum red-shift of $3 \mathrm{~nm}$ was obtained by applying an electrical power of $8.2 \mathrm{~mW}$ to the heater. No degradation of the responsivity or dark current was observed up to this point. Although the amount of tuning is still not sufficient to cover the whole FSR of the ring cavity, it can be used as a trimming method to compensate the wavelength drift from, e.g., fabrication errors.
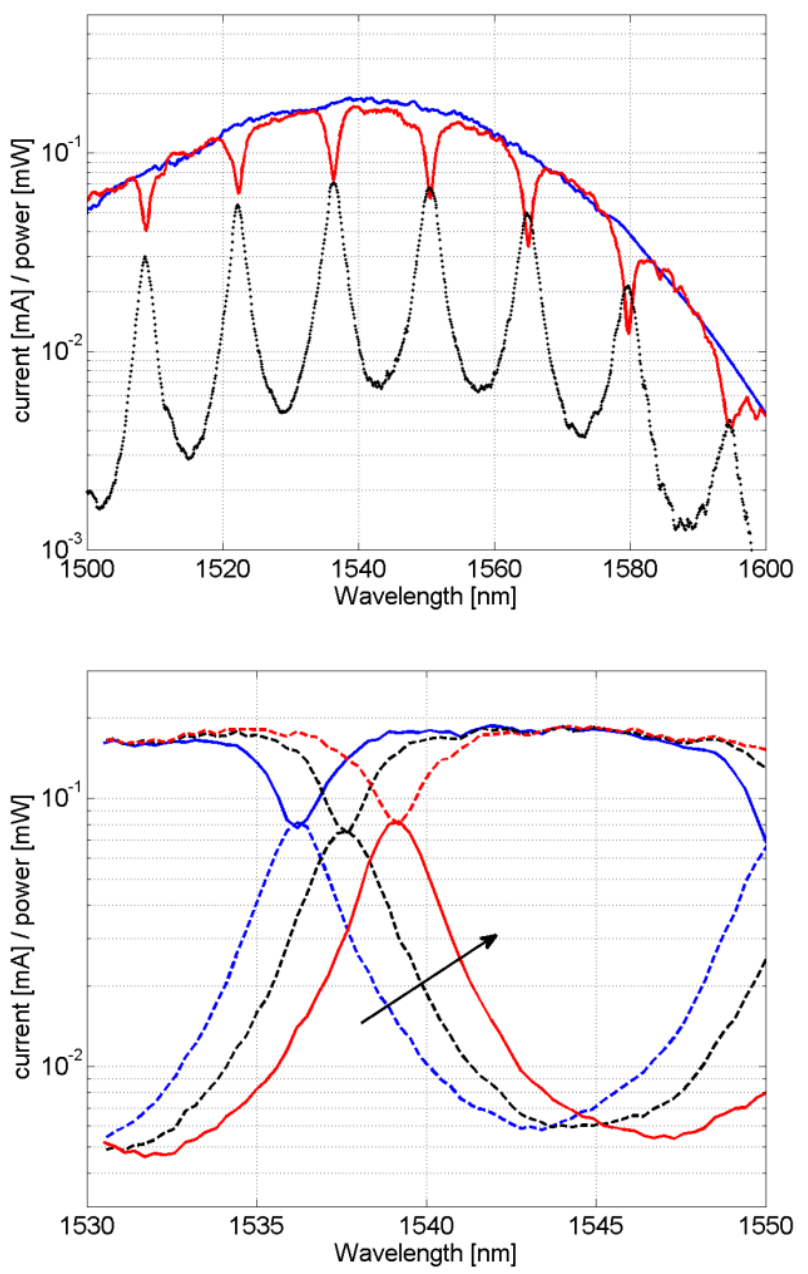

Fig. 22(a) Measured transmission spectrum and detected current of a fabricated device. Solid line indicates the incident power spectrum; dashed lines indicate the transmission spectrum; dotted lines indicate the detected current. Since grating couplers were used to interface to the access fibers, the input light follows a Gaussian spectrum (b) Transmission and current at different electrical powers applied to the heater. Along the arrow direction, the applied powers are $0 \mathrm{~mW}, 4.2 \mathrm{~mW}$, and $8.2 \mathrm{~mW}$, respectively.. 


\section{CONCLUSION}

We described the design and operation of a microdisk laser, fabricated using heterogeneous operation and its possible application in compact optical networks. Through improved design and processing, the threshold current could be decreased to $350 \mu \mathrm{A}$ and the output power under $\mathrm{CW}$ operation increased to $120 \mu \mathrm{W}$. The latter in turn allowed to demonstrate more advanced functionality, needed for scaling up the optical network capacity, such as all-optical wavelength conversion at high speed and optical flip-flop operation. Next we discussed the passive waveguide circuit fabrication process, its robustness versus fabrication variations and the implementation of thermal tuning to overcome these variations. Finally we proposed a tunable resonant detector, which may become an essential component in wavelength routed networks.

\section{REFERENCES}

[1] F. E. Doany, C. L. Schow, C. W. Baks, D. A. Kuchta, P. Pepeljugoski, L. Schares, R. Budd, F. Libsch, R. Dangel, F. Horst, B. J. Offrein, and J. A. Kash, "160 Gb/s Bidirectional Polymer-Waveguide Board-Level Optical Interconnects Using CMOS-Based Transceivers," Ieee Transactions on Advanced Packaging, vol. 32, pp. 345-359, May 2009.

[2] A. Suzuki, Y. Wakazono, T. Ishikawa, Y. Hashimoto, H. Masuda, S. Suzuki, M. Tamura, T. Suzuki, K. Kikuchi, H. Nakagawa, M. Aoyagi, and T. Mikawa, "Low-Cost Optical Subassembly Using VCSEL Pre-Self-Aligned With Optical Fiber for Optical Interconnect Applications," Journal of Lightwave Technology, vol. 27, pp. 4516-4523, Oct 2009.

[3] Y. A. Vlasov and S. J. McNab, "Losses in single-mode silicon-on-insulator strip waveguides and bends," Optics Express, vol. 12, pp. 1622-1631, Apr 2004.

[4] W. Bogaerts, S. Selvaraja, P. Dumon, J. Brouckaert, K. De Vos, D. Van Thourhout, and R. Baets, " Silicon-on-Insulator Spectral Filters Fabricated with CMOS Technology," J. Sel. Top. Quantum Electron, p. Accepted for publication, 2009

[5] T. Tsuchizawa, K. Yamada, H. Fukuda, T. Watanabe, T. Jun-ichi, M. Takahashi, T. Shoji, E. Tamechika, S. Itabashi, and H. Morita, "Microphotonics devices based on silicon microfabrication technology," Selected Topics in Quantum Electronics, IEEE Journal of, vol. 11, pp. 232-240, 2005.

[6] M. Lipson, "Compact electro-optic modulator's on a silicon chip," Ieee Journal of Selected Topics in Quantum Electronics, vol. 12, pp. 1520-1526, Nov-Dec 2006.

[7] W. M. J. Green, M. J. Rooks, L. Sekaric, and Y. A. Vlasov, "Ultra-compact, low RF power, $10 \mathrm{gb} / \mathrm{s}$ silicon Mach-Zehnder modulator," Optics Express, vol. 15, pp. 17106-17113, Dec 2007.

[8] D. Marris-Morini, L. Vivien, G. Rasigade, J. M. Fedeli, E. Cassan, X. Le Roux, P. Crozat, S. Maine, A. Lupu, P. Lyan, P. Rivallin, M. Halbwax, and S. Laval, "Recent Progress in High-Speed Silicon-Based Optical Modulators," Proceedings of the Ieee, vol. 97, pp. 1199-1215, Jul 2009.

[9] L. Vivien, J. Osmond, J. M. Fedeli, D. Marris-Morini, P. Crozat, J. F. Damlencourt, E. Cassan, Y. Lecunff, and S. Laval, "42 GHz p.i.n Germanium photodetector integrated in a silicon-on-insulator waveguide," Optics Express, vol. 17, pp. 6252-6257, Apr 2009.

[10]L. Chen, K. Preston, S. Manipatruni, and M. Lipson, "Integrated GHz silicon photonic interconnect with micrometer-scale modulators and detectors," Optics Express, vol. 17, pp. 15248-15256, Aug 2009.

[11]O. Liboiron-Ladouceur, H. Wang, A. S. Garg, and K. Bergman, "Low-power, transparent optical network interface for high bandwidth off-chip interconnects," Optics Express, vol. 17, pp. 6550-6561, Apr 2009.

[12]A. V. Krishnamoorthy, R. Ho, X. Z. Zheng, H. Schwetman, J. Lexau, P. Koka, G. L. Li, I. Shubin, and J. E. Cunningham, "Computer Systems Based on Silicon Photonic Interconnects," Proceedings of the Ieee, vol. 97, pp. 1337-1361, Jul 2009.

[13] J. Ahn, M. Fiorentino, R. G. Beausoleil, N. Binkert, A. Davis, D. Fattal, N. P. Jouppi, M. McLaren, C. M. Santori, R. S. Schreiber, S. M. Spillane, D. Vantrease, and Q. Xu, "Devices and architectures for photonic chip-scale integration," Applied Physics a-Materials Science \& Processing, vol. 95, pp. 989-997, Jun 2009.
[14]D. A. B. Miller, "Device Requirements for Optical Interconnects to Silicon Chips," Proceedings of the Ieee, vol. 97, pp. 1166-1185, Jul 2009.

[15]W. D. Zhong and R. S. Tucker, "Wavelength routing-based photonic packet buffers and their applications in photonic packet switching systems," Journal of Lightwave Technology, vol. 16, pp. 1737-1745, 1998.

[16] T. Mitze, M. Schnarrenberger, L. Zimmermann, J. Bruns, F. Fidorra, K. Janiak, J. Kreissl, S. Fidorra, H. Heidrich, and K. Petermann, "CWDM transmitter module based on hybrid integration," Ieee Journal of Selected Topics in Quantum Electronics, vol. 12, pp. 983-987, Sep-Oct 2006.

[17] C. G. Fonstad, J. J. Rumpler, E. R. Barkley, J. M. Perkins, and S. Famenini, "Recess integration of micro-cleaved laser diode platelets with dielectric waveguides on silicon - art. no. 69090O," in Conference on Novel in-Plane Semiconductor Lasers VII, San Jose, CA, 2008, pp. O9090-O9090.

[18] N. Jokerst, M. Royal, S. Palit, L. Luan, S. Dhar, and T. Tyler, "Chip scale integrated microresonator sensing systems," Journal of Biophotonics, vol. 2, pp. 212-226, Apr 2009.

[19]G. Roelkens, J. Brouckaert, D. Taillaert, P. Dumon, W. Bogaerts, D. Van Thourhout, R. Baets, R. Notzel, and M. Smit, "Integration of InP/InGaAsP photodetectors onto silicon-on-insulator waveguide circuits," Optics Express, vol. 13, pp. 10102-10108, Dec 122005.

[20] A. W. Fang, M. N. Sysak, B. R. Koch, R. Jones, E. Lively, Y. H. Kuo, D. Liang, O. Raday, and J. E. Bowers, "Single-Wavelength Silicon Evanescent Lasers," Ieee Journal of Selected Topics in Quantum Electronics, vol. 15, pp. 535-544, May-Jun 2009.

[21]T. Okumura, T. Maruyama, M. Kanemaru, S. Sakamoto, and S. Arai, "Single-mode operation of GaInAsP/InP-membrane distributed feedback lasers bonded on silicon-on-insulator substrate with rib-waveguide structure," Japanese Journal of Applied Physics Part 2-Letters \& Express Letters, vol. 46, pp. L1206-L1208, Dec 2007.

[22]D. Liang, J. E. Bowers, D. C. Oakley, A. Napoleone, D. C. Chapman, C. L. Chen, P. W. Juodawlkis, and O. Raday, "High-Quality $150 \mathrm{~mm}$ InP-to-Silicon Epitaxial Transfer for Silicon Photonic Integrated Circuits," Electrochemical and Solid State Letters, vol. 12, pp. H101-H104, 2009.

[23] M. Kostrzewa, L. Di Cioccio, M. Zussy, J. C. Roussin, J. M. Fedeli, N. Kernevez, P. Regreny, C. Lagahe-Blanchard, and B. Aspar, "InP dies transferred onto silicon substrate for optical interconnects application," Sensors and Actuators a-Physical, vol. 125, pp. 411-414, Jan 2006.

[24]G. Roelkens, J. Brouckaert, D. Van Thourhout, R. Baets, R. Notzel, and M. Smit, "Adhesive bonding of InP/InGaAsP dies to processed silicon-on-insulator wafers using DVS-bis-benzocyclobutene," Journal of the Electrochemical Society, vol. 153, pp. G1015-G1019, 2006.

[25] S. L. McCall, A. F. J. Levi, R. E. Slusher, S. J. Pearton, and R. A. Logan, "WHISPERING-GALLERY MODE MICRODISK LASERS," Applied Physics Letters, vol. 60, pp. 289-291, 1992.

[26]C. Seassal, P. Rojo-Romeo, X. Letartre, P. Viktorovitch, G. Hollinger, E. Jalaguier, S. Pocas, and B. Aspar, "InP microdisk lasers on silicon wafer: $\mathrm{CW}$ room temperature operation at $1.6 \mathrm{mu}$ m," Electronics Letters, vol. 37, pp. 222-223, Feb 2001.

[27]H. T. Hattori, C. Seassal, E. Touraille, P. Rojo-Romeo, X. Letartre, G. Hollinger, P. Viktorovitch, L. Di Cioccio, M. Zussy, L. El Melhaoui, and J. M. Fedeli, "Heterogeneous integration of microdisk lasers on silicon strip waveguides for optical interconnects," Ieee Photonics Technology Letters, vol. 18, pp. 223-225, Jan-Feb 2006.

[28]P. R. Romeo, J. Van Campenhout, P. Regreny, A. Kazmierczak, C. Seassal, X. Letartre, G. Hollinger, D. Van Thourhout, R. Baets, M. Fedeli, and L. Di Cioccio, "Heterogeneous integration of electrically driven microdisk based laser sources for optical interconnects and photonic ICs," Optics Express, vol. 14, pp. 3864-3871, May 2006.

[29] J. Van Campenhout, P. Rojo-Romeo, P. Regreny, C. Seassal, D. Van Thourhout, S. Verstuyft, L. Di Cioccio, J. M. Fedeli, C. Lagahe, and R. Baets, "Electrically pumped InP-based microdisk lasers integrated with a nanophotonic silicon-on-insulator waveguide circuit," Optics Express, vol. 15, pp. 6744-6749, May 2007.

[30] S. K. Selvaraja, P. Jaenen, W. Bogaerts, D. Van Thourhout, P. Dumon, and R. Baets, "Fabrication of Photonic Wire and Crystal Circuits in Silicon-on-Insulator Using 193-nm Optical Lithography," Journal of Lightwave Technology, vol. 27, pp. 4076-4083, Sep 2009.

[31]F. Mandorlo, P. Rojo-Romeo, X. Letartre, and P. Viktorovitch, "A simple perturbative analysis for fast design of an electrically pumped micro-disk laser," Optics Express, vol. 17, pp. 70-79, Jan 2009.

[32] J. E. Heebner, T. C. Bond, and J. S. Kallman, "Generalized formulation for performance degradations due to bending and edge scattering loss in microdisk resonators," Optics Express, vol. 15, pp. 4452-4473, Apr 2007.

[33] J. Van Campenhout, P. Rojo-Romeo, D. Van Thourhout, C. Seassal, P. Regreny, L. Di Cioccio, J. M. Fedeli, and R. Baets, "Thermal 
characterization of electrically injected thin-film InGaAsP microdisk lasers on Si," Journal of Lightwave Technology, vol. 25, pp. 1543-1548, Jun 2007.

[34]D. Taillaert, F. Van Laere, M. Ayre, W. Bogaerts, D. Van Thourhout, P. Bienstman, and R. Baets, "Grating couplers for coupling between optical fibers and nanophotonic waveguides," Japanese Journal of Applied Physics Part 1-Regular Papers Brief Communications \& Review Papers, vol. 45, pp. 6071-6077, Aug 2006.

[35]D. A. B. Miller, "Device requirements for optical interconnects to silicon chips," Proc. IEEE Special issue on silicon photonics, 2009.

[36] J. Van Campenhout, L. Liu, P. R. Romeo, D. Van Thourhout, C. Seassal, P. Regreny, L. Di Cioccio, J. M. Fedeli, and R. Baets, "A compact SOI-integrated multiwavelength laser source based on cascaded InP microdisks," Ieee Photonics Technology Letters, vol. 20, pp. 1345-1347, Jul-Aug 2008.

[37]L. Liu, G. Roelkens, J. Van Campenhout, J. Brouckaert, D. Van Thourhout, and R. Baets, "III-V/silicon-on-insulator nanophotonic cavities for optical network-on-chip," Journal of Nanoscience and Nanotechnology (Invited), accepted for publication, 2010.

[38]F. Mandorlo, P. Rojo-Romeo, X. Letarte, J. M. Fedeli, and P. Viktorovitch, "Improvement of threshold and mode selectivity of a microdisk laser by engineering its coupling to an external passive cavity," in Group IV Photonics San Fransisco, 2009.

[39]C. Manolatou, M. J. Khan, S. H. Fan, P. R. Villeneuve, H. A. Haus, and J. D. Joannopoulos, "Coupling of modes analysis of resonant channel add-drop filters," Ieee Journal of Quantum Electronics, vol. 35, pp. 1322-1331, Sep 1999.

[40]L. Liu, J. Van Campenhout, G. Roelkens, D. Van Thourhout, P. Rojo-Romeo, P. Regreny, C. Seassal, J. M. Fedeli, and R. Baets, "Ultralow-power all-optical wavelength conversion in a silicon-on-insulator waveguide based on a heterogeneously integrated III-V microdisk laser," Applied Physics Letters, vol. 93, p. 3, Aug 2008.

[41] O. Raz, L. Liu, D. Van Thourhout, P. Rojo-Romeo, J. M. Fedeli, and H. J. S. Dorren, "High Speed Wavelength Conversion in a Heterogeneously Integrated Disc Laser Over Silicon On Insulator for Network on a Chip Applications," in European Conference on Optical Communications, Vienna, Austria, 2009, p. 4.2.3.

[42]M. Sorel, P. J. R. Laybourn, G. Giuliani, and S. Donati, "Unidirectional bistability in semiconductor waveguide ring lasers," Applied Physics Letters, vol. 80, pp. 3051-3053, Apr 2002.

[43]M. Sorel, G. Giuliani, A. Scire, R. Miglierina, S. Donati, and P. J. R. Laybourn, "Operating regimes of GaAs-AlGaAs semiconductor ring lasers: Experiment and model," Ieee Journal of Quantum Electronics, vol. 39, pp. 1187-1195, Oct 2003.

[44]L. Liu, R. Kumar, K. Huybrechts, T. Spuesens, G. Roelkens, E.-J. Geluk, T. de Vries, P. Regreny, D. Van Thourhout, R. Baets, and G. Morthier, "An ultra-small low power all-optical flip-flop memory on a silicon chip," nature photonics (accepted), 2010.

[45] S. K. Selvaraja, P. Jaenen, W. Bogaerts, D. Van Thourhout, P. Dumon, and R. G. Baets, "Fabrication of Photonic Wire and Crystal Circuits in Silicon-on-Insulator Using 193-nm Optical Lithography," Lightwave Technology, Journal of, vol. 27, pp. 4076-4083, 2009.

[46]S. K. Selvaraja, P. Jaenen, S. Beckx, W. Bogaert, P. Dumon, D. Van Thourout, and R. Bates, "Silicon nanophotonic wire structures fabricated by $193 \mathrm{~nm}$ optical lithography," in Lasers and Electro-Optics Society, 2007. LEOS 2007. The 20th Annual Meeting of the IEEE, 2007, pp. 48-49.

[47] S. K. Selvaraja, W. Bogaerts, P. Dumon, D. Van Thourhout, and R. Baets, "Sub-nanometer Linewidth Uniformity in Silicon Nano-photonic Waveguide Devices Using CMOS Fabrication Technology," Journal of Selected Topics in Quantum Electronics, DOI 10.1109/JSTQE.2009.2026550, 2009.

[48]F. N. Xia, M. Rooks, L. Sekaric, and Y. Vlasov, "Ultra-compact high order ring resonator filters using submicron silicon photonic wires for on-chip optical interconnects," Optics Express, vol. 15, pp. 11934-11941, Sep 17 2007.

[49]F. N. Xia, L. Sekaric, and Y. Vlasov, "Ultracompact optical buffers on a silicon chip," Nature Photonics, vol. 1, pp. 65-71, Jan 2007.

[50]F. Gan, T. Barwicz, M. A. Popovic, M. S. Dahlem, C. W. Holzwarth, P. T. Rakich, H. I. Smith, E. P. Ippen, F. X. Kartner, and Ieee, "Maximizing the thermo-optic tuning range of silicon photonic structures," in Photonics in Switching 2007 Conference, San Francisco, CA, 2007, pp. 67-68.

[51]I. Christiaens, D. Van Thourhout, and R. Baets, "Low-power thermo-optic tuning of vertically coupled microring resonators," Electronics Letters, vol. 40, pp. 560-561, Apr 2004.
[52]L. Liu, J. Brouckaert, G. Roelkens, D. Van Thourhout, and R. Baets, " Compact, Wavelength-Selective Resonant Photodetector Based on III-V/Silicon-on-Insulator Heterogeneous Integration," in Conference on Lasers and Electro-Optics/International Quantum Electronics Conference (CLEO) Baltimore, 2009, p. paper CTuV3.

[53] J. Brouckaert, G. Roelkens, D. Van Thourhout, and R. Baets, "Thin-film III-V photodetectors integrated on silicon-on-insulator photonic ICs," Journal of Lightwave Technology, vol. 25, pp. 1053-1060, Apr 2007.

\section{Biographies}

Dries Van Thourhout (M'98) received the degree in physical engineering and the Ph.D. degree from Ghent University, Ghent, Belgium in 1995 and 2000, respectively. From October 2000 to September 2002, he was with Lucent Technologies, Bell Laboratories, Crawford Hill, NJ, working on InPInGaAsP monolithically integrated devices. In October 2002, he joined the Department of Information Technology (INTEC), Ghent University, continuing his work on integrated optoelectronic devices. Main interests are heterogeneous integration by wafer bonding, intrachipoptical interconnect and WDM-devices.

T. Spuesens (M'08) was born in Sas van Gent, The Netherlands, in 1982. He received the M.Sc. degree in electrical engineering from Eindhoven University of Technology, Eindhoven, The Netherlands, in 2008. In August 2008 he joined the Photonics Research Group at INTEC, Ghent University, Ghent, Belgium, where he is currently working towards his Ph.D. degree in photonics.

His current research interests are in the field of heterogeneous integration of Silicon-on-Insulator and III-V material, optical interconnects and microsources. He is involved in the FP7 project WADIMOS.

Shankar Kumar Selvaraja (S'06) received the M.Tech. degree in Optical communication from College of engineering, Anna University, Chennai, India, in 2004, the M.Sc. degree in Microsystems and Microelectronics from University of Twente, The Netherlands, in 2005. Since 2006 he is working towards his $\mathrm{PhD}$ at Photonic research group of IMEC-Ghent University, Belgium. His research interest includes CMOS compatible process development for Photonic integrated circuits: optical lithography (193 and 248 $\mathrm{nm}$ ), dry etch and deposition process. He is a student member of IEEE and IEEE photonics society.

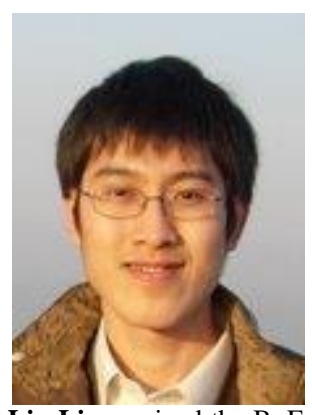

Liu Liu received the B. Eng. in Information Engineering in 2002 at Zhejiang University, China, and Ph.D. in Photonics in 2006 at the Royal Institute of Technology (KTH), Sweden. He joined Photonics Research Group, Department of Information Technology (INTEC), Ghent University, Belgium, as a post doctoral researcher from 2007 to 2009. Now he is with Department of Photonics Engineering, Technical University of Denmark, DTU - Fotonik, Denmark. His current research area is heterogeneous integration, and silicon nanophotonic devices. 


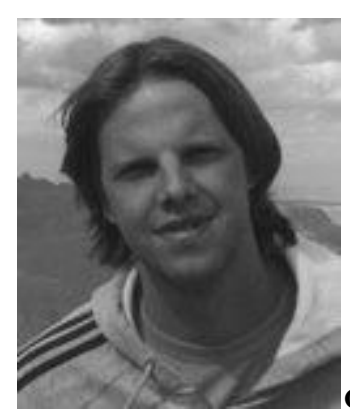

Günther Roelkens (S'02-M'07) was born in 1979 in Ghent, Belgium. He graduated in 2002 as an electronics engineer from Ghent University (with highest honour). His M.S. thesis was entitled "Ring resonators for photonic IC's based on III-V semiconductors," for which he received the Barco/FWO award. Since 2002, he has been working in the Photonics Research Group at Ghent University, where he received the doctoral degree in April 2007, for his work in the field of heterogeneous III-V/Silicon photonics. Currently, he is also appointed assistant professor at the Technical University Eindhoven, The Netherlands.

Rajesh Kumar received 'Bachelor of Science and Bachelor of Education'(Physical Science) from Regional Institute of Education(NCERT), Ajmer, India in 2004. He received Master of Science(Physic) and Master of Technology(Optoelectronics and Optical Communication) from Indian Institute of Technology Delhi, New Delhi, India, In 2006 and 2008 respectively. Previously he worked on metal-oxide semiconductor thin films. His current area of research is microdisk based photonic components and devices for optical communication. He is student member of OSA and SPIE.

Geert Morthier e received the degree in electrical engineering and the Ph.D. degree from Ghent University in 1987 and 1991 respectively. Since 1991 he is a member of the permanent staff of IMEC and since 2001 he is parttime professor at Ghent University. His main interests are in the modelling and characterisation of optoelectronic and photonic components. He has authored or co-authored over 150 papers in the field and holds several patents. $\mathrm{He}$ is currently the project manager of the EU-HISTORIC project, which focuses on heterogeneously integrated microdisk lasers for gating and flip-flops and their combination into photonic integrated circuits. He has been involved in research on DFB laser modelling, on widely tunable lasers and their control and on all-optical signal processing. His current research interest is mainly on different types of all-optical flip-flops for optical packet switching.

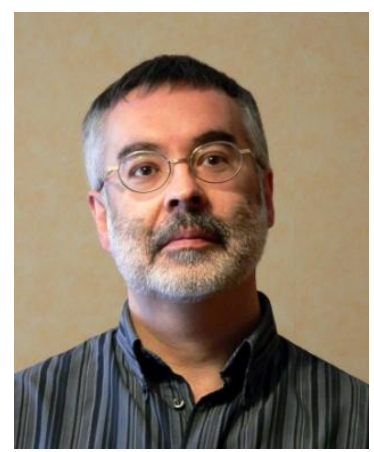

P. Rojo Romeo was born in 1958 in Madrid (Spain). He received the InG. Dipl. degree in physics in 1981 from the Institut National des Sciences Appliquées (INSA) in Lyon, France, and the Ph.D. degree in Electronic devices in 1984 at INSA (Lyon). He is currently an Associate Professor (since 1988) in electronics at Ecole Centrale de Lyon (France), at the Institute of Nanotechnologies of Lyon (INL). His primary research interests include electrical and optical devices fabrication technology, characterization of micro-electronic and opto-electronic micro - nano - devices. He is also involved in optical interconnection systems, nanotechnologies, and optical integrated circuits technologies.

In his recent research he focused on heterogeneous integration of III-V structures on $\mathrm{Si}$ - based substrates, development of CMOS compatible III-V nanolasers coupled to $\mathrm{Si}$ waveguides, development of novel 2.5D photonic crystal lasers for guided and free space laser emission, localization of nanostructures on a patterned surface using e-beam lithography techniques : localization of InAs quantum dots on InP for single photon sources, localized growth of III-V nanowires on $\mathrm{Si}$, localization of metal nanostructures for plasmonic applications.

Philippe Regreny was born in France in 1967. He received is degree in materials science from Rennes University, Rennes, France in 1991 and PhD in electronics from Ecole centrale de lyon, Lyon, France, in 1997.

Since 1998 he is a research engineer at INL/CNRS, Ecole Centrale de Lyon, Ecully, France. His current research interests concern epitaxial growth of III-V semiconductors and oxide materials and their integration on silicon. He is author and co-author of 80 publications.

Mandorlo Fabien received his engineer diploma from the Ecole Centrale de Lyon, Ecully, France in 2005, and the same year his M.S degree in electronics from the University of Lyon. After three years as a Ph.D student at both the Institute of Nanotechnologies of Lyon (INL) and the Commissariat a l'Energie Atomique/Laboratoire d'Electronique de Technologie de l'Information (CEA/LETI), Grenoble, he is actually a teaching assistant. Main activities concerns microlasers and their integration in CMOS processes for optical integration in micro-electronics.

Oded Raz (S'05, M'07) was born in the Netherlands in 1970, and has been living in Israel since 1973. He received his B.Sc. degree in Electrical engineering from the Technion, Israel's Institute of Technology, Haifa, Israel in 1993 and his M.Sc. and PhD from the Tel Aviv University, Tel Aviv Israel in 2002 and 2007 respectively. Since 2007 he is working as a researcher in the COBRA research institute at the Technical University of Eindhoven, The Netherlands, where he is now an assistant professor. His main research fields are all optical signal processing for high speed data signals, integrated III-V

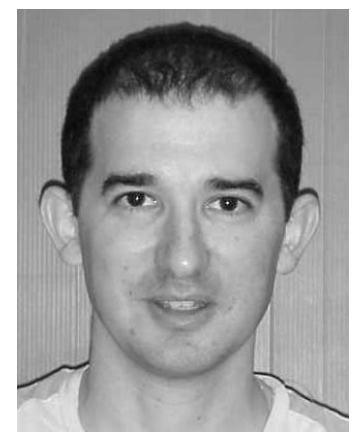
optical circuits, SOI circuits and heterogeneous integration of InP over SOI for digital photonics. His other fields of interest include optical communications systems and microwave-photonics.

Christophe KOPP received his $\mathrm{PhD}$ degree in photonic engineering from the University of Strasbourg in 2000 for his works on diffractive optics. He has joined the CEA/LETI in 2001 to develop assembling techniques for micro-optoelectronic devices. His research interests are focused today on high speed optical data transmission applications, integrated silicon photonics design, imaging systems.

Laurent Grenouillet received the Engineer degree in physics in 1998 from the INSA (National Institute of Applied Sciences) in Lyon, France, and the Ph.D. degree from INSA Lyon, France, in 2001 for his work on the optical spectroscopy of diluted nitrides grown on GaAs substrates. After a post doctoral position at CEA-Leti (Electronics and Information Technology Lab), Grenoble, France in the field of Molecular Beam Epitaxy, he joined the Optronics Department at CEA-Leti where he was in charge of the MBE growth

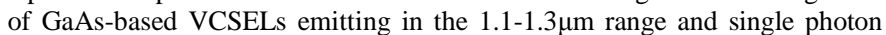
sources. Since 2006, he has been involved in the field of Silicon Photonics. Main interests are heterogeneous integration of III-Vs on silicon, CMOS compatible hybrid laser sources. 